\title{
Natural language processing algorithms for mapping clinical text fragments onto ontology concepts: a systematic review and recommendations for future studies
}

Martijn G. Kersloot ${ }^{1,2^{*}}$ (D) Florentien J. P. van Putten ${ }^{1}$, Ameen Abu-Hanna ${ }^{1}$, Ronald Cornet ${ }^{1}$ and Derk L. Arts ${ }^{1,2}$

\begin{abstract}
Background: Free-text descriptions in electronic health records (EHRs) can be of interest for clinical research and care optimization. However, free text cannot be readily interpreted by a computer and, therefore, has limited value. Natural Language Processing (NLP) algorithms can make free text machine-interpretable by attaching ontology concepts to it. However, implementations of NLP algorithms are not evaluated consistently. Therefore, the objective of this study was to review the current methods used for developing and evaluating NLP algorithms that map clinical text fragments onto ontology concepts. To standardize the evaluation of algorithms and reduce heterogeneity between studies, we propose a list of recommendations.

Methods: Two reviewers examined publications indexed by Scopus, IEEE, MEDLINE, EMBASE, the ACM Digital Library, and the ACL Anthology. Publications reporting on NLP for mapping clinical text from EHRs to ontology concepts were included. Year, country, setting, objective, evaluation and validation methods, NLP algorithms, terminology systems, dataset size and language, performance measures, reference standard, generalizability, operational use, and source code availability were extracted. The studies' objectives were categorized by way of induction. These results were used to define recommendations.

Results: Two thousand three hundred fifty five unique studies were identified. Two hundred fifty six studies reported on the development of NLP algorithms for mapping free text to ontology concepts. Seventy-seven described development and evaluation. Twenty-two studies did not perform a validation on unseen data and 68 studies did not perform external validation. Of 23 studies that claimed that their algorithm was generalizable, 5 tested this by external validation. A list of sixteen recommendations regarding the usage of NLP systems and algorithms, usage of data, evaluation and validation, presentation of results, and generalizability of results was developed.

(Continued on next page)
\end{abstract}

\footnotetext{
* Correspondence: m.g.kersloot@amsterdamumc.nl

${ }^{1}$ Amsterdam UMC, University of Amsterdam, Department of Medical Informatics, Amsterdam Public Health Research Institute Castor EDC, Room J1B-109, PO Box 22700, 1100 DE Amsterdam, The Netherlands

${ }^{2}$ Castor EDC, Amsterdam, The Netherlands
}

C C The Author(s). 2020 Open Access This article is licensed under a Creative Commons Attribution 4.0 International License, which permits use, sharing, adaptation, distribution and reproduction in any medium or format, as long as you give appropriate credit to the original author(s) and the source, provide a link to the Creative Commons licence, and indicate if changes were made. The images or other third party material in this article are included in the article's Creative Commons licence, unless indicated otherwise in a credit line to the material. If material is not included in the article's Creative Commons licence and your intended use is not permitted by statutory regulation or exceeds the permitted use, you will need to obtain permission directly from the copyright holder. To view a copy of this licence, visit http://creativecommons.org/licenses/by/4.0/. The Creative Commons Public Domain Dedication waiver (http://creativecommons.org/publicdomain/zero/1.0/) applies to the data made available in this article, unless otherwise stated in a credit line to the data. 


\begin{abstract}
(Continued from previous page)
Conclusion: We found many heterogeneous approaches to the reporting on the development and evaluation of NLP algorithms that map clinical text to ontology concepts. Over one-fourth of the identified publications did not perform an evaluation. In addition, over one-fourth of the included studies did not perform a validation, and 88\% did not perform external validation. We believe that our recommendations, alongside an existing reporting standard, will increase the reproducibility and reusability of future studies and NLP algorithms in medicine.
\end{abstract}

Keywords: Ontologies, Entity linking, Annotation, Concept mapping, Named-entity recognition, Natural language processing, Evaluation studies, Recommendations for future studies

\section{Background}

One of the main activities of clinicians, besides providing direct patient care, is documenting care in the electronic health record (EHR). Currently, clinicians document clinical findings and symptoms primarily as free-text descriptions within clinical notes in the EHR since they are not able to fully express complex clinical findings and nuances of every patient in a structured format $[1,2]$. These freetext descriptions are, amongst other purposes, of interest for clinical research $[3,4]$, as they cover more information about patients than structured EHR data [5]. However, free-text descriptions cannot be readily processed by a computer and, therefore, have limited value in research and care optimization.

One method to make free text machine-processable is entity linking, also known as annotation, i.e., mapping free-text phrases to ontology concepts that express the phrases' meaning. Ontologies are explicit formal specifications of the concepts in a domain and relations among them [6]. In the medical domain, SNOMED CT [7] and the Human Phenotype Ontology (HPO) [8] are examples of widely used ontologies to annotate clinical data. After the data has been annotated, it can be reused by clinicians to query EHRs $[9,10]$, to classify patients into different risk groups $[11,12]$, to detect a patient's eligibility for clinical trials [13], and for clinical research [14].

Natural Language Processing (NLP) can be used to (semi-)automatically process free text. The literature indicates that NLP algorithms have been broadly adopted and implemented in the field of medicine $[15,16]$, including algorithms that map clinical text to ontology concepts [17]. Unfortunately, implementations of these algorithms are not being evaluated consistently or according to a predefined framework and limited availability of data sets and tools hampers external validation [18].

To improve and standardize the development and evaluation of NLP algorithms, a good practice guideline for evaluating NLP implementations is desirable $[19,20]$. Such a guideline would enable researchers to reduce the heterogeneity between the evaluation methodology and reporting of their studies. Generic reporting guidelines such as TRIPOD [21] for prediction models, STROBE [22] for observational studies, RECORD [23] for studies conducted using routinely-collected health data, and STARD [24] for diagnostic accuracy studies, are available, but are often not used in NLP research. This is presumably because some guideline elements do not apply to NLP and some NLP-related elements are missing or unclear. We, therefore, believe that a list of recommendations for the evaluation methods of and reporting on NLP studies, complementary to the generic reporting guidelines, will help to improve the quality of future studies.

In this study, we will systematically review the current state of the development and evaluation of NLP algorithms that map clinical text onto ontology concepts, in order to quantify the heterogeneity of methodologies used. We will propose a structured list of recommendations, which is harmonized from existing standards and based on the outcomes of the review, to support the systematic evaluation of the algorithms in future studies.

\section{Methods}

This study consists of two phases: a systematic review of the literature and the formation of recommendations based on the findings of the review.

\section{Literature review}

A systematic review of the literature was performed using the Preferred Reporting Items for Systematic reviews and Meta-Analyses (PRISMA) statement [25].

\section{Search strategy and study selection}

We searched Scopus, IEEE, MEDLINE, EMBASE, the Association for Computing Machinery (ACM) Digital Library, and the Association for Computational Linguistics (ACL) Anthology for the following keywords: Natural Language Processing, Medical Language Processing, Electronic Health Record, reports, charts, clinical notes, clinical text, medical notes, ontolog", concept", encod", annotat", code, and coding. We excluded the words 'reports' and 'charts' in the ACL and ACM databases since these databases also contain publications on non-medical subjects. The detailed search strategies for each database can be found in Additional file 2. We searched until December 19, 2019 and applied the 
filters "English" and "has abstract" for all databases. Moreover, we applied the filters "Medicine, Health Professions, and Nursing" for Scopus, the filters "Conferences", "Journals", and "Early Access Articles" for IEEE, and the filter "Article" for Scopus and EMBASE. EndNote X9 [26] and Rayyan [27] were used to review and delete duplicates.

The selection process consisted of three phases. In the first phase, two independent reviewers with a Medical Informatics background (MK, FP) individually assessed the resulting titles and abstracts and selected publications that fitted the criteria described below.

Inclusion criteria were:

- Medical language processing as the main topic of the publication

- Use of EHR data, clinical reports, or clinical notes

- Algorithm performs annotation

- Publication is written in English

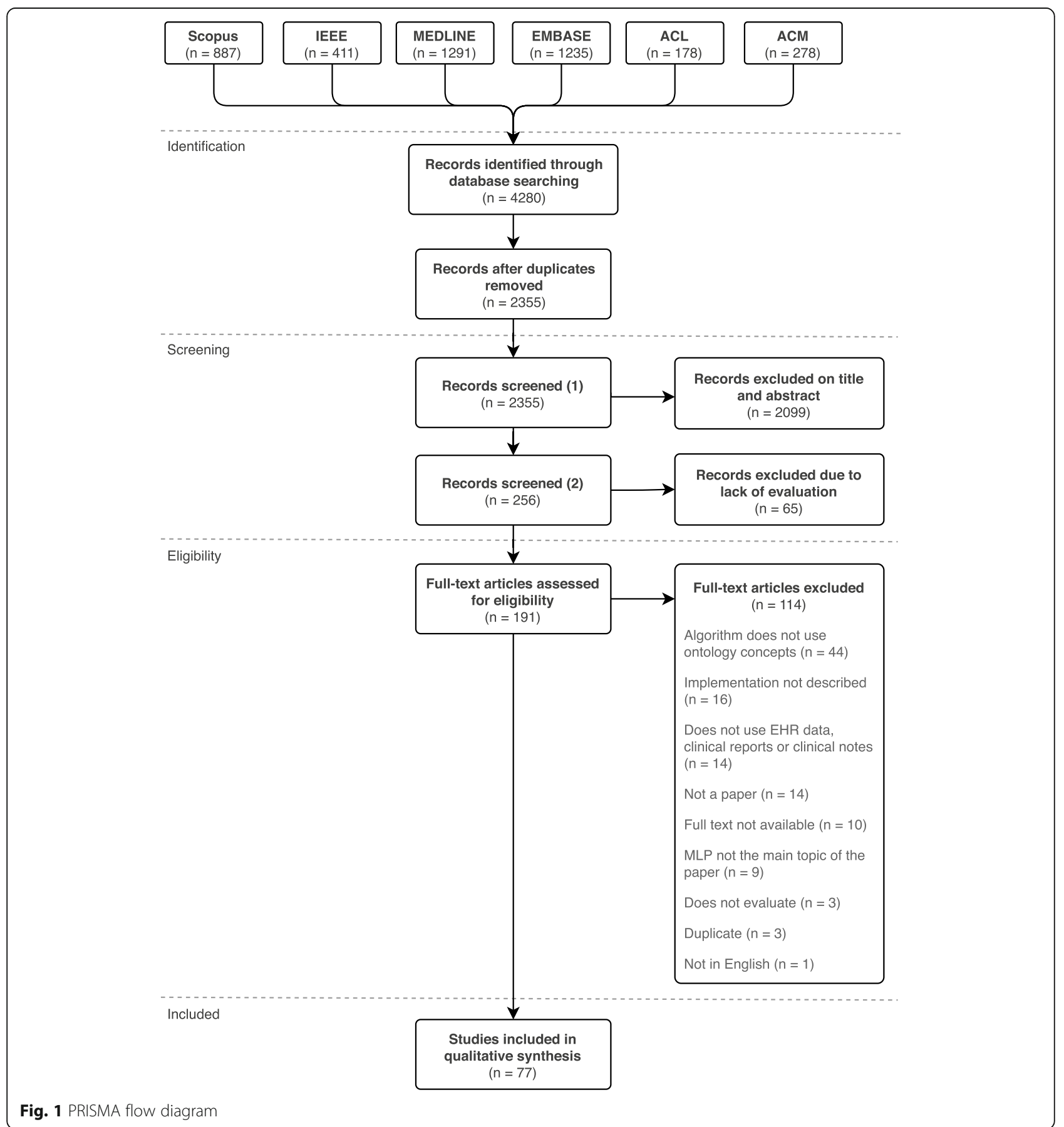


Table 1 Induced objective tasks with their definition and an example

\begin{tabular}{|c|c|c|}
\hline Induced NLP task(s) & Description & Example \\
\hline Concept detection $^{1}$ & $\begin{array}{l}\text { Assign ontology concepts to phrases in free } \\
\text { text (i.e., entity linking or annotation) }\end{array}$ & $\begin{array}{l}\text { "Systolic blood pressure" can be represented as SNOMED-CT } \\
\text { concept } 271649006 \text { | Systolic blood pressure (observable entity) | }\end{array}$ \\
\hline Event detection & Detect events in free text & $\begin{array}{l}\text { "Patient visited the outpatient clinic in January } 2020 \text { " is an } \\
\text { event of type Visit. }\end{array}$ \\
\hline Relationship detection & $\begin{array}{l}\text { Detect semantic relationships between } \\
\text { concepts in free text }\end{array}$ & $\begin{array}{l}\text { The concept Lung cancer in "This patient was diagnosed with } \\
\text { recurrent lung cancer" is related to the concept Recurrence. }\end{array}$ \\
\hline Text normalization & $\begin{array}{l}\text { Transform free text into a single canonical } \\
\text { form }\end{array}$ & $\begin{array}{l}\text { "This patient was diagnosed with influenza last year." becomes } \\
\text { "This patient be diagnose with influenza last year." }\end{array}$ \\
\hline Text summarization & $\begin{array}{l}\text { Create a short summary of free text and } \\
\text { possible restructure the text based on this } \\
\text { summary }\end{array}$ & $\begin{array}{l}\text { "Last year, this patient visited the clinic and was diagnosed with } \\
\text { diabetes mellitus type 2, and in addition to his diabetes, the } \\
\text { patient was also diagnosed with hypertension" becomes } \\
\text { "Last year, this patient was diagnosed with diabetes mellitus } \\
\text { type } 2 \text { and hypertension". }\end{array}$ \\
\hline Classification & Assign categories to free text & $\begin{array}{l}\text { A report containing the text "This patient is not diagnosed } \\
\text { yet" will be assigned to the category Undiagnosed. }\end{array}$ \\
\hline Prediction & Create a predictive model based on free text & $\begin{array}{l}\text { Predict the outcome of the APACHE score based on the } \\
\text { (free-text) content in a patient chart. }\end{array}$ \\
\hline Identification & $\begin{array}{l}\text { Identify documents (e.g., reports or patient } \\
\text { charts) that match a specific condition } \\
\text { based on the contents of the document }\end{array}$ & $\begin{array}{l}\text { Find all patient charts that describe patients with hypertension } \\
\text { and a BMl above } 30 \text {. }\end{array}$ \\
\hline Software development & $\begin{array}{l}\text { Develop new or build upon existing NLP } \\
\text { software }\end{array}$ & $\begin{array}{l}\text { A new algorithm was developed to map ontology concepts } \\
\text { to free text in clinical reports. }\end{array}$ \\
\hline Software evaluation & Evaluate the effectiveness of NLP software & The mapping algorithm has an F-score of 0.874 . \\
\hline
\end{tabular}

${ }^{1}$ Also known as Medical Entity Linking and Medical Concept Normalization

Some studies do not describe the application of NLP in their study by only listing NLP as the used method, instead of describing its specific implementation. Additionally, some studies create their own ontology to perform NLP tasks, instead of using an established, domain-accepted ontology. Both approaches limit the generalizability of the study's methods. Therefore, we defined the following exclusion criteria:

- Implementation was not described

- Implementation does not use an existing established ontology for encoding

Table 2 Induced objective categories with their definition and associated NLP task(s)

\begin{tabular}{|c|c|c|}
\hline Induced category & Induced NLP task(s) & Definition \\
\hline Computer-assisted coding & Concept detection & Perform semi-automated annotation (i.e., with a human in the loop) \\
\hline Information comparison & $\begin{array}{l}\text { Concept detection } \\
\text { Event detection } \\
\text { Relationship detection }\end{array}$ & Compare extracted structured information to information available in free-text form \\
\hline Information enrichment & $\begin{array}{l}\text { Concept detection } \\
\text { Event detection } \\
\text { Relationship detection } \\
\text { Text normalization } \\
\text { Text summarization }\end{array}$ & Extract structured information from free text and attach this new information to the source \\
\hline Information extraction & $\begin{array}{l}\text { Concept detection } \\
\text { Event detection } \\
\text { Relationship detection }\end{array}$ & Extract structured information from free text \\
\hline Prediction & $\begin{array}{l}\text { Classification } \\
\text { Prediction } \\
\text { Identification }\end{array}$ & Use structured information to classify free-text reports, predict outcomes, or identify cases \\
\hline $\begin{array}{l}\text { Software development } \\
\text { and evaluation }\end{array}$ & $\begin{array}{l}\text { Software development } \\
\text { Software evaluation }\end{array}$ & Develop new NLP software or evaluate new or existing NLP software \\
\hline Text processing & $\begin{array}{l}\text { Text normalization } \\
\text { Text summarization }\end{array}$ & Transform free text into a new, more comprehensible form \\
\hline
\end{tabular}




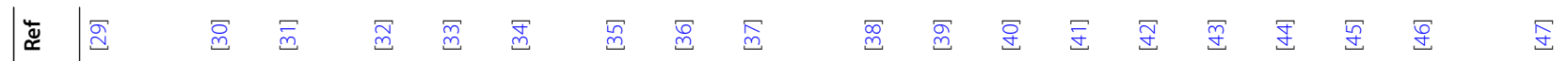

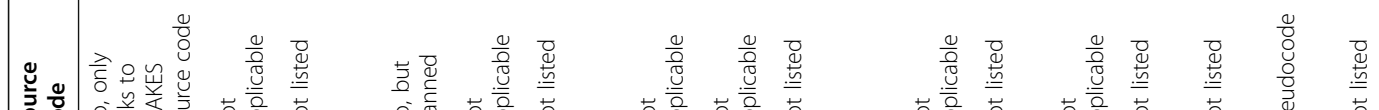

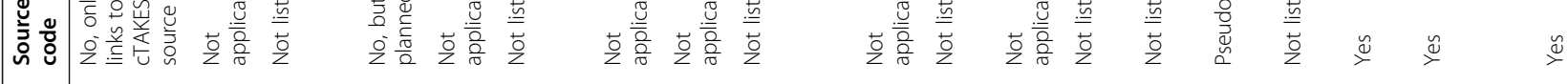

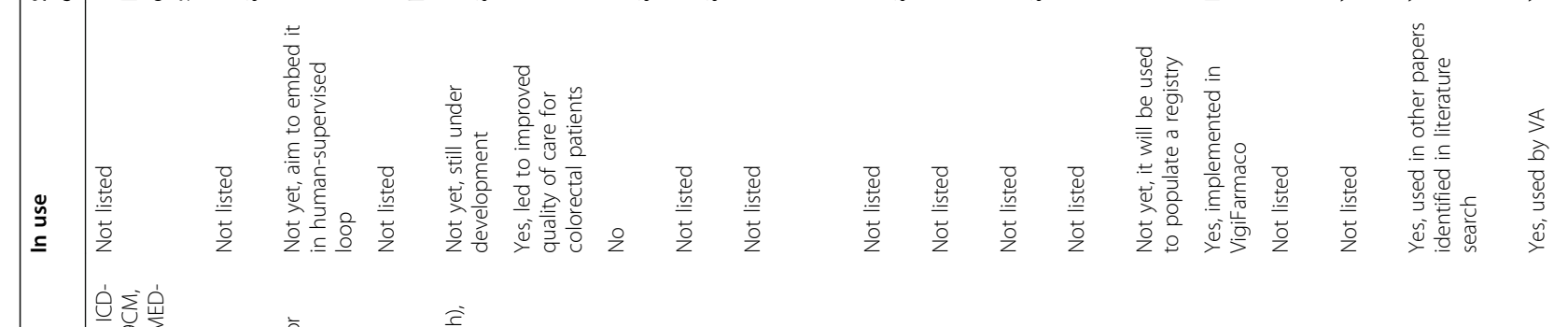
它究崖

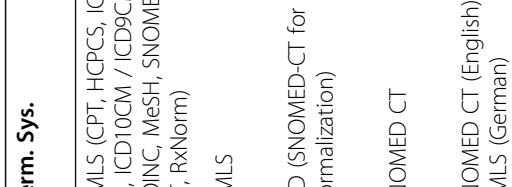

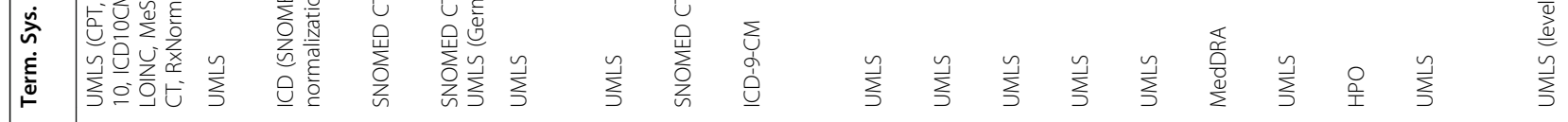

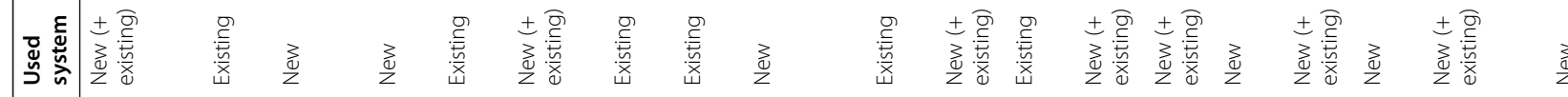

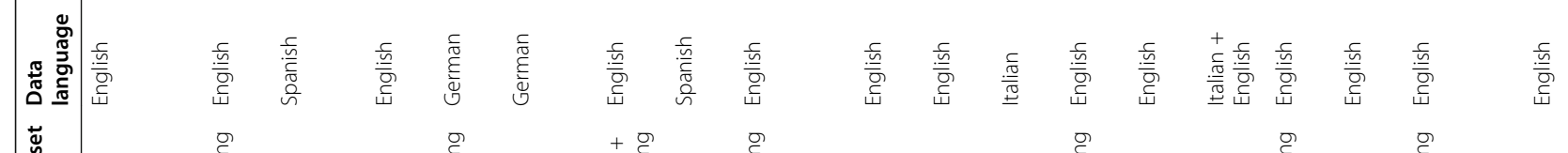

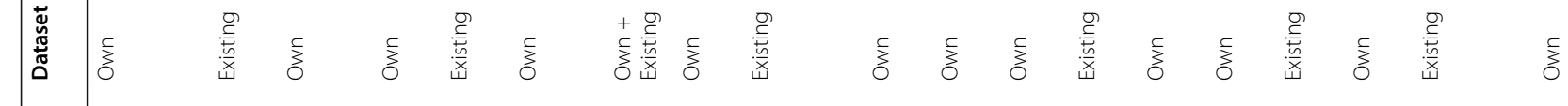

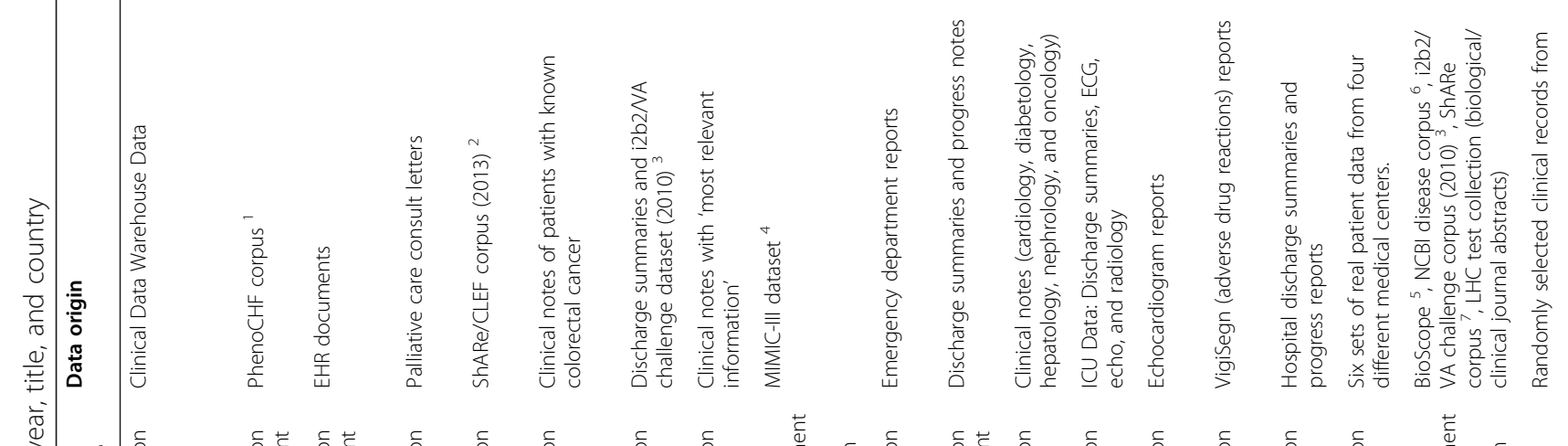

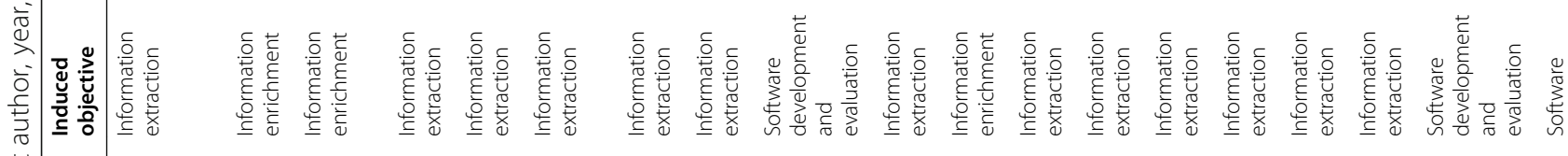

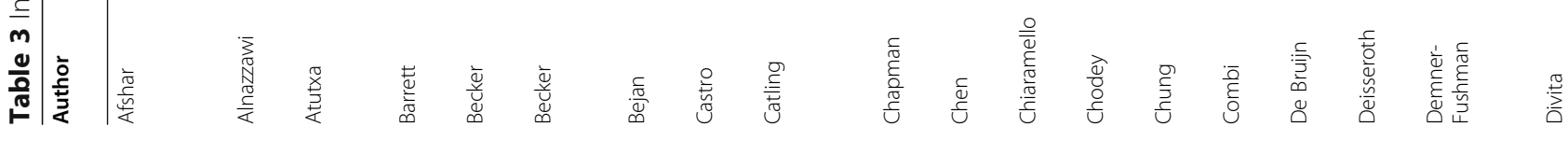




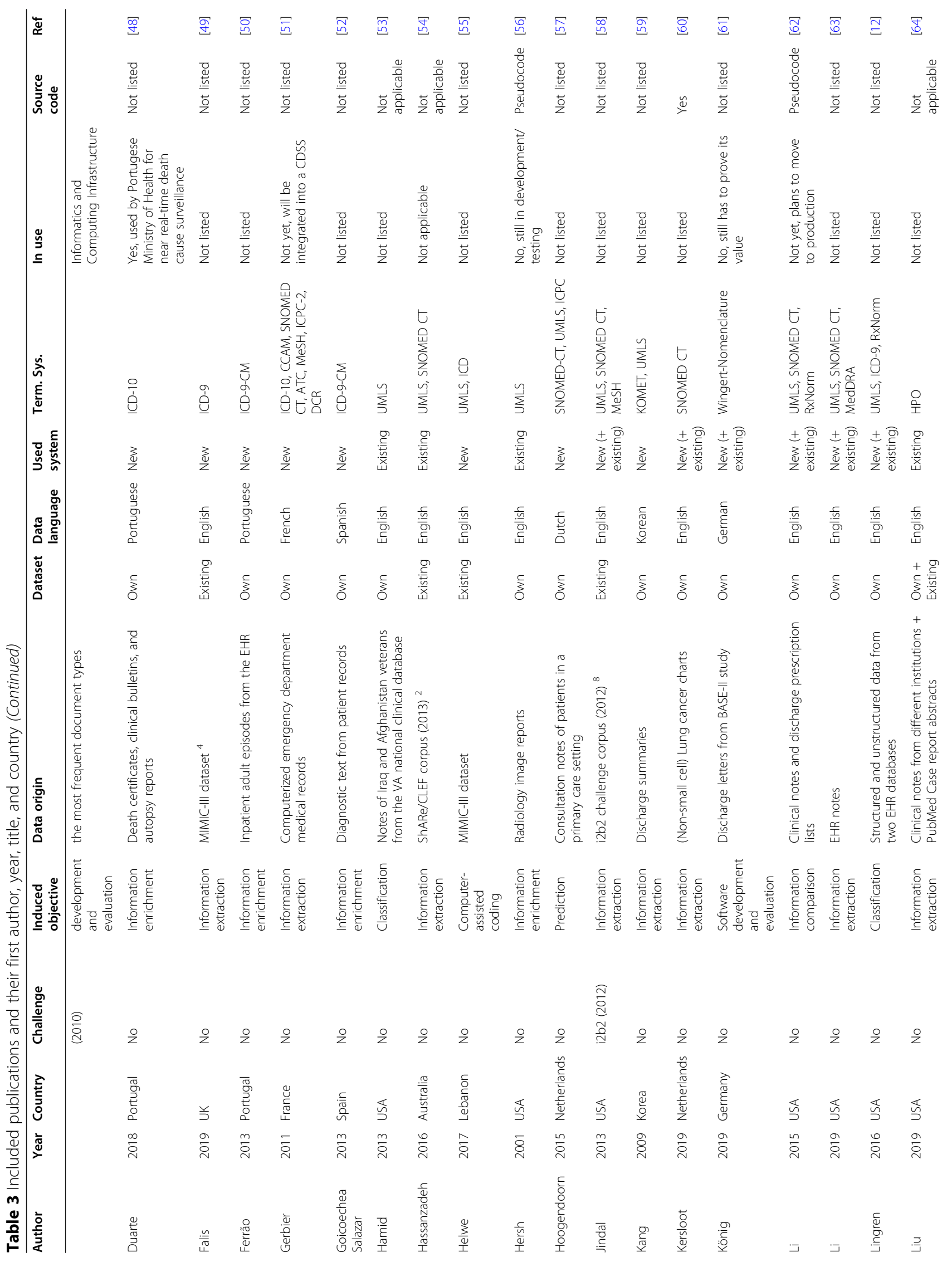




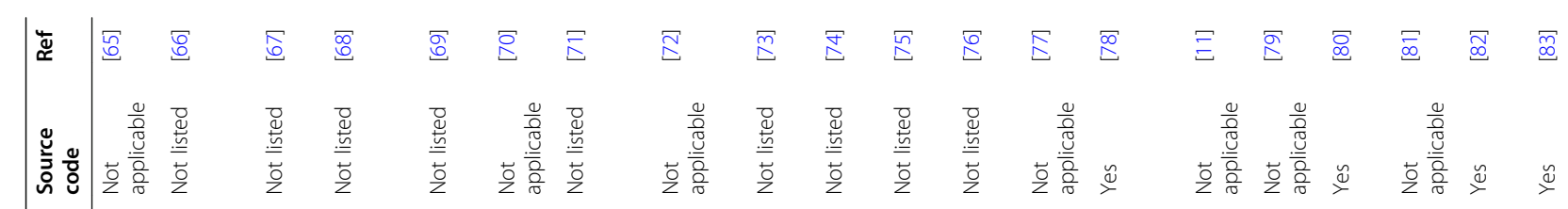

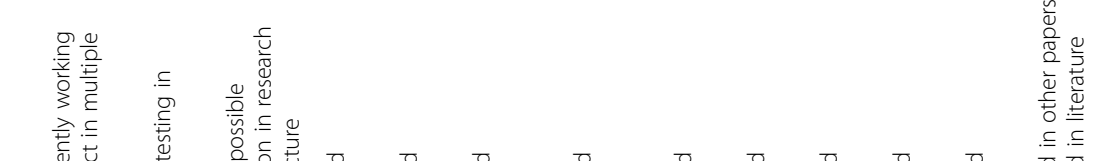

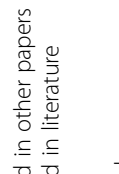

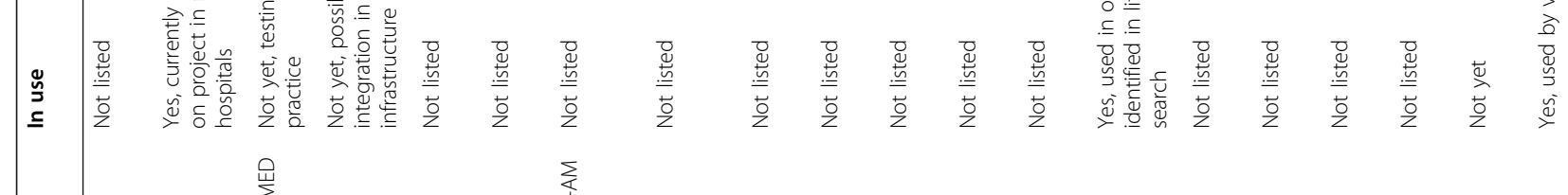

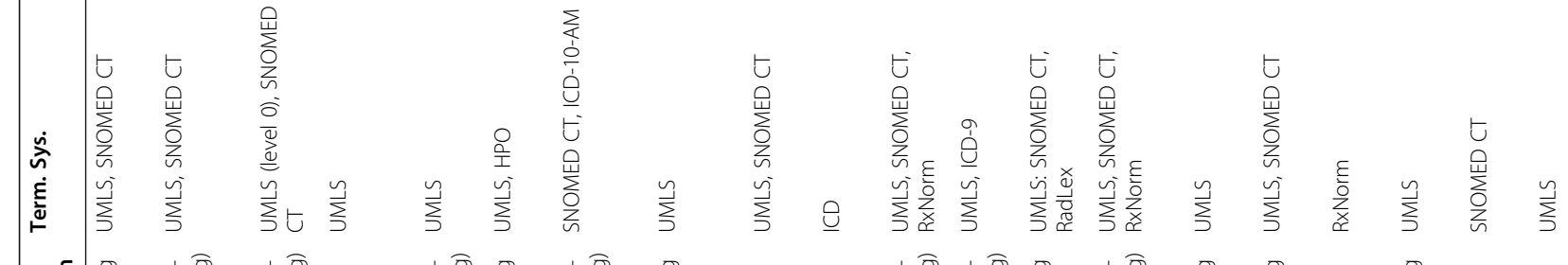

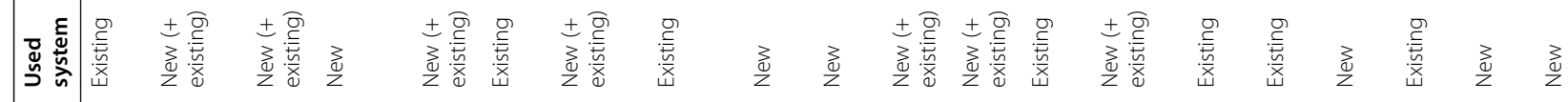

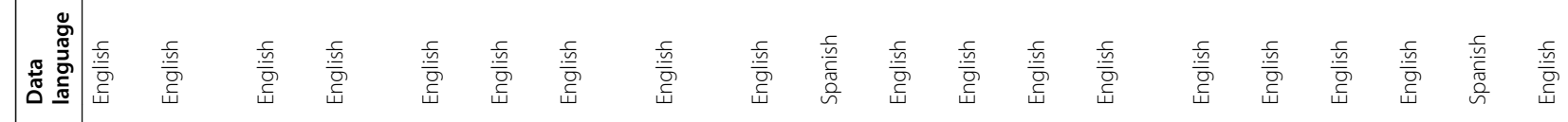

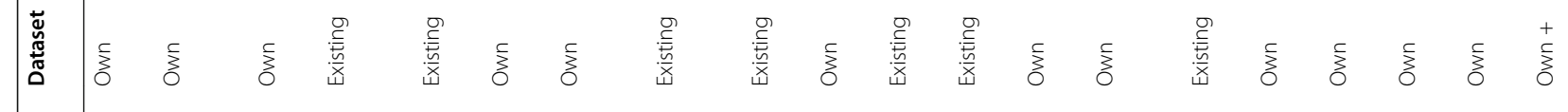

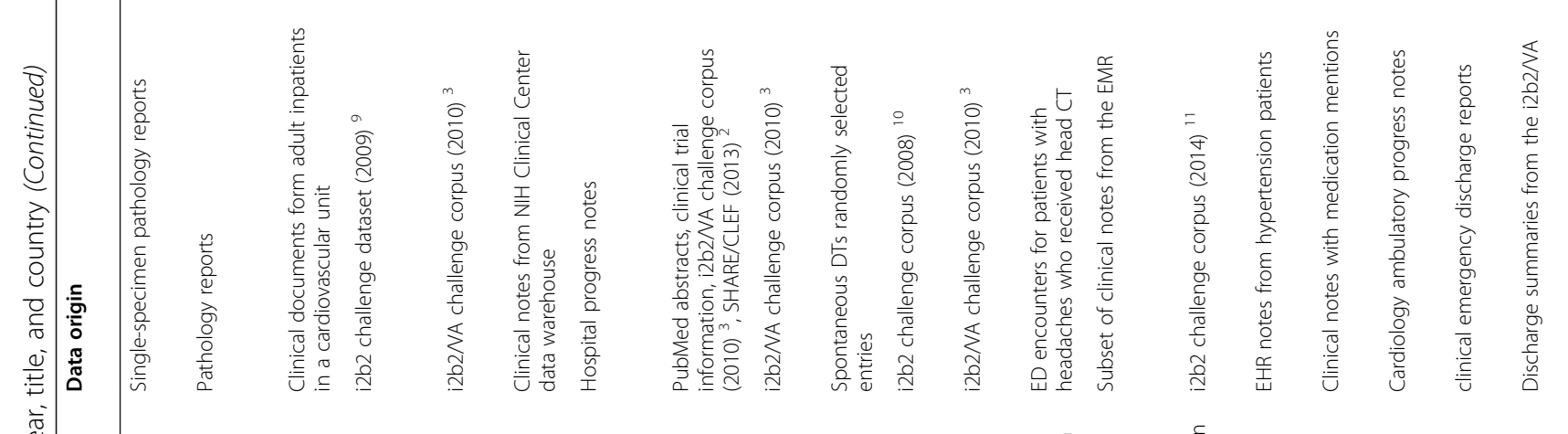

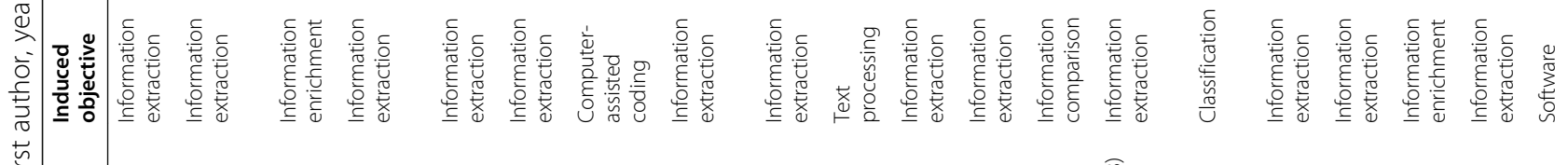

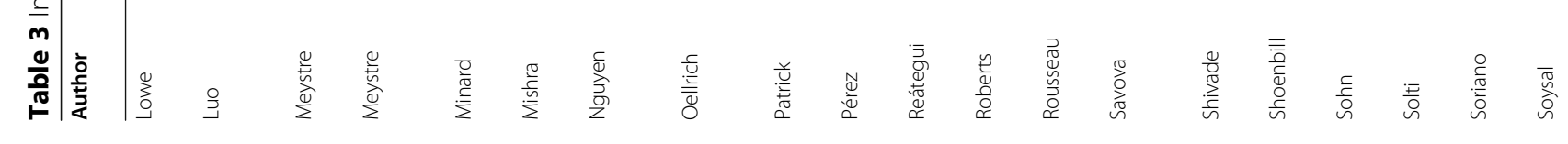




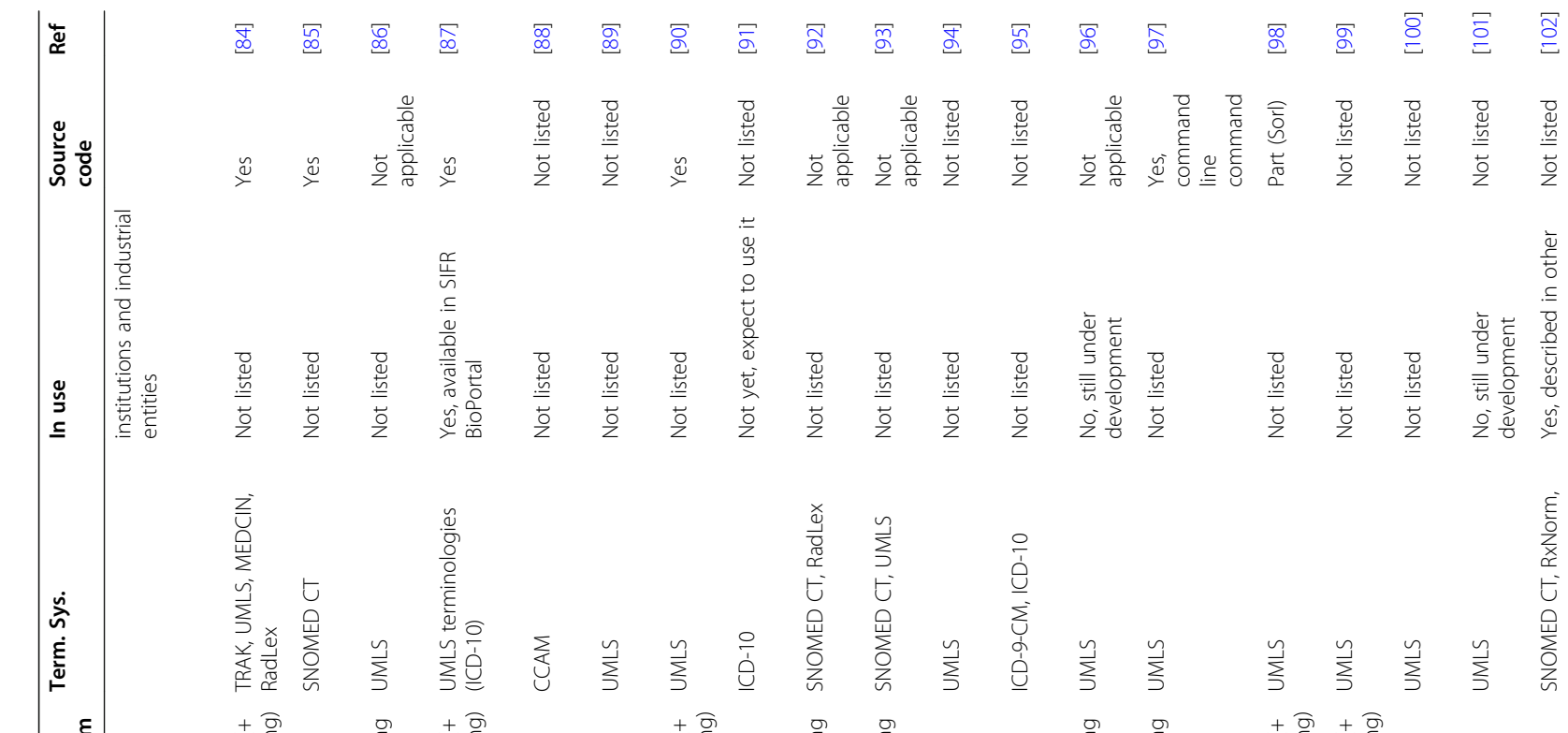

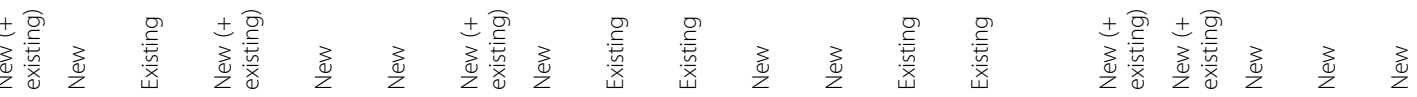

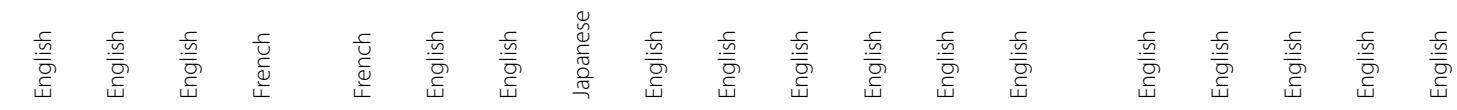

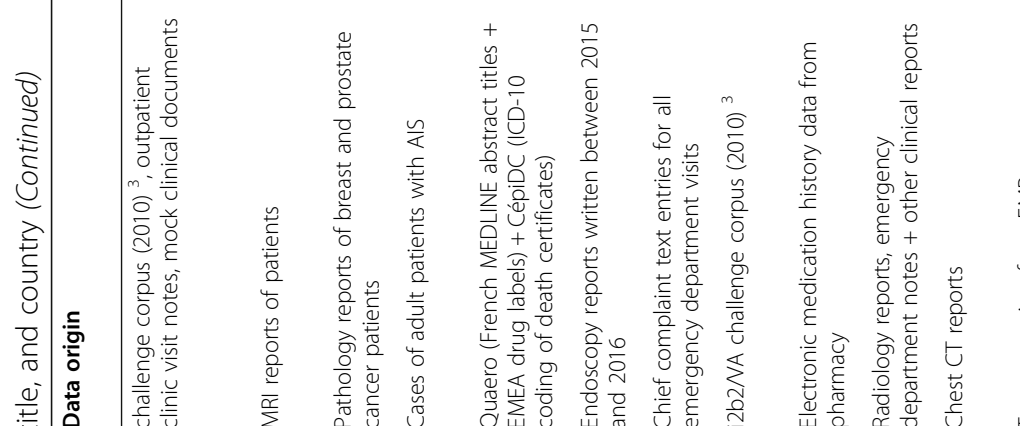




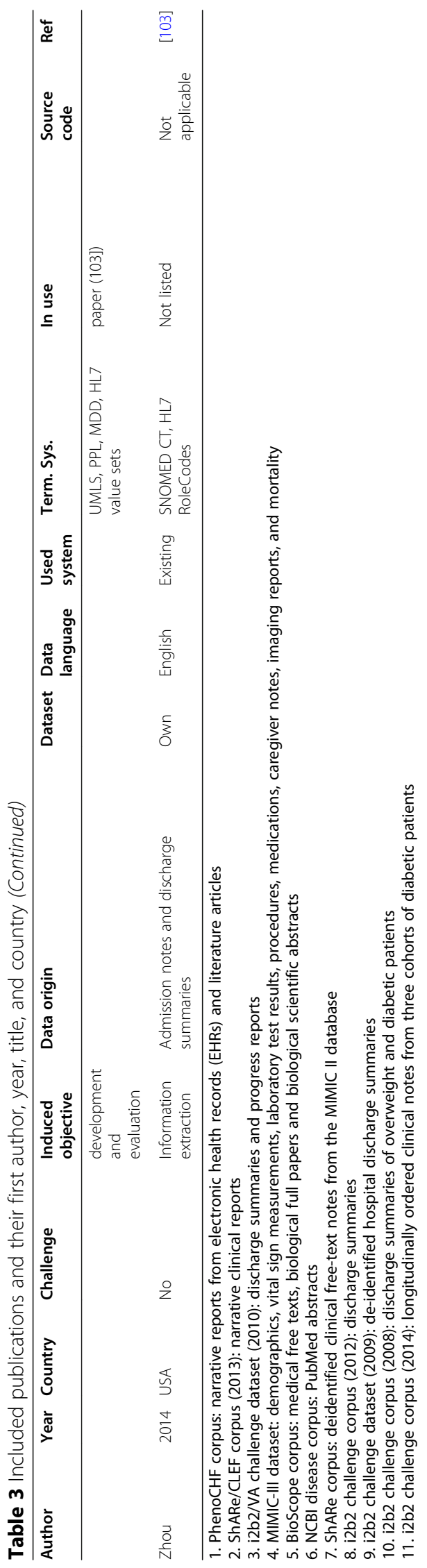


Table 4 Included publications and their evaluation methodologies

\begin{tabular}{|c|c|c|c|c|c|c|}
\hline Author & Year & Ref. std. & Validation & External & Generalizability $^{a}$ & Ref \\
\hline Afshar & 2019 & $\begin{array}{l}\text { Existing EHR } \\
\text { data }\end{array}$ & $\begin{array}{l}\text { Hold-out validation (train, } \\
\text { test, development) }\end{array}$ & No & No, validation is needed & [29] \\
\hline Alnazzawi & 2016 & $\begin{array}{l}\text { Existing } \\
\text { annotated } \\
\text { corpus }\end{array}$ & External & $\begin{array}{l}\text { ShARe/CLEF, NCBI disease, } \\
\text { Heart failure and pulmonary } \\
\text { embolism corpora }\end{array}$ & $\begin{array}{l}\text { Yes, achieves competitive } \\
\text { performance on other corpora }\end{array}$ & [30] \\
\hline Atutxa & 2018 & $\begin{array}{l}\text { Manual } \\
\text { retrospective } \\
\text { review }\end{array}$ & $\begin{array}{l}\text { Hold-out validation (train, } \\
\text { test, development) }\end{array}$ & No & $\begin{array}{l}\text { Yes, easily portable to other } \\
\text { languages }\end{array}$ & [31] \\
\hline Barrett & 2013 & $\begin{array}{l}\text { Manual } \\
\text { annotations }\end{array}$ & 10-fold cross validation & $\begin{array}{l}\text { Multiple datasets (different } \\
\text { provider) }\end{array}$ & Yes, expect that it is generalizable & [32] \\
\hline Becker & 2016 & $\begin{array}{l}\text { Existing } \\
\text { annotated } \\
\text { corpus }\end{array}$ & Not used & No & Not listed & [33] \\
\hline Becker & 2019 & $\begin{array}{l}\text { Manual } \\
\text { annotations }\end{array}$ & $\begin{array}{l}\text { Hold-out validation (train, } \\
\text { test, development) }\end{array}$ & No & Not listed & [34] \\
\hline Bejan & 2015 & $\begin{array}{l}\text { Manual } \\
\text { annotations }\end{array}$ & External & i2b2 data (2010) & $\begin{array}{l}\text { Yes, good performance on the } \\
\text { i2b2 dataset, even though not } \\
\text { optimized on it }\end{array}$ & [35] \\
\hline Castro & 2010 & $\begin{array}{l}\text { Manual } \\
\text { annotations }\end{array}$ & Not used & No & Not listed & [36] \\
\hline Catling & 2018 & $\begin{array}{l}\text { Existing } \\
\text { annotated } \\
\text { corpus }\end{array}$ & $\begin{array}{l}\text { Hold-out validation (train, } \\
\text { test, development) }\end{array}$ & No & Not listed & [37] \\
\hline Chapman & 2004 & $\begin{array}{l}\text { Manual } \\
\text { annotations }\end{array}$ & Not used & No & $\begin{array}{l}\text { Yes, generalizable to other domains } \\
\text { within and outside of bio surveillance }\end{array}$ & [38] \\
\hline Chen & 2016 & $\begin{array}{l}\text { Manual } \\
\text { annotations }\end{array}$ & 10-fold cross validation & No & Not listed & [39] \\
\hline Chiaramello & 2016 & $\begin{array}{l}\text { Manual } \\
\text { annotations }\end{array}$ & Not used & No & Not listed & [40] \\
\hline Chodey & 2016 & $\begin{array}{l}\text { Existing } \\
\text { annotated } \\
\text { corpus }\end{array}$ & $\begin{array}{l}\text { Hold-out validation (train, } \\
\text { test) }\end{array}$ & No & Not listed & [41] \\
\hline Chung & 2005 & $\begin{array}{l}\text { Manual } \\
\text { annotations }\end{array}$ & $\begin{array}{l}\text { Hold-out validation (train, } \\
\text { test) }\end{array}$ & $\begin{array}{l}\text { Reports from a second } \\
\text { hospital }\end{array}$ & Not listed & [42] \\
\hline Combi & 2018 & $\begin{array}{l}\text { Manual } \\
\text { annotations }\end{array}$ & Not used & No & Not listed & [43] \\
\hline deBruijn & 2011 & $\begin{array}{l}\text { Existing } \\
\text { annotated } \\
\text { corpus }\end{array}$ & 15 -fold cross validation & No & Not listed & [44] \\
\hline Deisseroth & 2019 & $\begin{array}{l}\text { Manual } \\
\text { annotations }\end{array}$ & $\begin{array}{l}\text { Hold-out validation (train, } \\
\text { test) }\end{array}$ & $\begin{array}{l}\text { Data from a second } \\
\text { hospital }\end{array}$ & $\begin{array}{l}\text { Yes, it can be immediately incorporated } \\
\text { into clinical practice }\end{array}$ & [45] \\
\hline $\begin{array}{l}\text { Demner- } \\
\text { Fushman }\end{array}$ & 2017 & $\begin{array}{l}\text { Existing } \\
\text { annotated } \\
\text { corpus }\end{array}$ & External & Multiple datasets & Not listed & [46] \\
\hline Divita & 2014 & $\begin{array}{l}\text { Manual } \\
\text { annotations }\end{array}$ & Not used & No & Not listed & [47] \\
\hline Duarte & 2018 & $\begin{array}{l}\text { Manual } \\
\text { annotations }\end{array}$ & $\begin{array}{l}\text { Hold-out validation (train, } \\
\text { test) }\end{array}$ & Second dataset & Not listed & [48] \\
\hline Falis & 2019 & $\begin{array}{l}\text { Existing } \\
\text { annotated } \\
\text { corpus }\end{array}$ & $\begin{array}{l}\text { Hold-out validation (train, } \\
\text { test, development) }\end{array}$ & No & $\begin{array}{l}\text { Yes, method is not specific to an } \\
\text { ontology, and could be used for a graph } \\
\text { of any formation }\end{array}$ & [49] \\
\hline Ferrão & 2013 & $\begin{array}{l}\text { Existing EHR } \\
\text { data }\end{array}$ & $\begin{array}{l}\text { Hold-out validation (train, } \\
\text { test) }\end{array}$ & No & Not listed & [50] \\
\hline Gerbier & 2011 & $\begin{array}{l}\text { Manual } \\
\text { annotations }\end{array}$ & $\begin{array}{l}\text { Hold-out validation (train, } \\
\text { test) }\end{array}$ & No & $\begin{array}{l}\text { Yes, it could also serve other types of } \\
\text { clinical decision support systems }\end{array}$ & [51] \\
\hline
\end{tabular}


Table 4 Included publications and their evaluation methodologies (Continued)

\begin{tabular}{|c|c|c|c|c|c|c|}
\hline Author & Year & Ref. std. & Validation & External & Generalizability $^{a}$ & Ref \\
\hline $\begin{array}{l}\text { Goicoechea } \\
\text { Salazar }\end{array}$ & 2013 & $\begin{array}{l}\text { Manual } \\
\text { annotations }\end{array}$ & $\begin{array}{l}\text { Hold-out validation (train, } \\
\text { test) }\end{array}$ & No & Not listed & [52] \\
\hline Hamid & 2013 & $\begin{array}{l}\text { Manual } \\
\text { annotations }\end{array}$ & 10-fold cross validation & No & $\begin{array}{l}\text { Possible, the classifier may be } \\
\text { applicable in academic hospital } \\
\text { samples }\end{array}$ & {$[53]$} \\
\hline Hassanzadeh & 2016 & $\begin{array}{l}\text { Existing } \\
\text { annotated } \\
\text { corpus }\end{array}$ & $\begin{array}{l}\text { Hold-out validation (train, } \\
\text { test) }\end{array}$ & No & Not applicable & {$[54]$} \\
\hline Helwe & 2017 & $\begin{array}{l}\text { Existing } \\
\text { annotated } \\
\text { corpus }\end{array}$ & $\begin{array}{l}\text { Hold-out validation (train, } \\
\text { test, development) }\end{array}$ & No & Not listed & {$[55]$} \\
\hline Hersh & 2001 & $\begin{array}{l}\text { Manual } \\
\text { annotations }\end{array}$ & $\begin{array}{l}\text { Hold-out validation (train, } \\
\text { test) }\end{array}$ & No & Not listed & {$[56]$} \\
\hline Hoogendoorn & 2015 & $\begin{array}{l}\text { Existing EHR } \\
\text { data }\end{array}$ & 5-fold cross validation & No & Not listed & [57] \\
\hline Jindal & 2013 & $\begin{array}{l}\text { Existing } \\
\text { annotated } \\
\text { corpus }\end{array}$ & $\begin{array}{l}\text { Hold-out validation (train, } \\
\text { test) }\end{array}$ & No & Yes, broad applicability & [58] \\
\hline Kang & 2009 & $\begin{array}{l}\text { Manual } \\
\text { annotations }\end{array}$ & $\begin{array}{l}\text { Hold-out validation (train, } \\
\text { test) }\end{array}$ & No & Yes, extensible to other languages & [59] \\
\hline Kersloot & 2019 & $\begin{array}{l}\text { Manual } \\
\text { annotations }\end{array}$ & $\begin{array}{l}\text { Hold-out validation } \\
\text { (development, test) }\end{array}$ & No & $\begin{array}{l}\text { Possible, but external validation } \\
\text { is needed }\end{array}$ & [60] \\
\hline König & 2019 & $\begin{array}{l}\text { Existing EHR } \\
\text { data }\end{array}$ & Not used & No & Still to be tested & [61] \\
\hline $\mathrm{Li}$ & 2015 & $\begin{array}{l}\text { Manual } \\
\text { annotations }\end{array}$ & 10-fold cross validation & No & Not listed & [62] \\
\hline Li & 2019 & $\begin{array}{l}\text { Existing } \\
\text { annotated } \\
\text { corpus }\end{array}$ & $\begin{array}{l}\text { Hold-out validation (train, } \\
\text { test, development) }\end{array}$ & No & Not listed & [63] \\
\hline Lingren & 2016 & $\begin{array}{l}\text { Manual } \\
\text { annotations }\end{array}$ & $\begin{array}{l}\text { Hold-out validation (train, } \\
\text { test, development) }\end{array}$ & No & Not listed & [12] \\
\hline Liu & 2019 & $\begin{array}{l}\text { Manual } \\
\text { annotations }\end{array}$ & Not used & $\begin{array}{l}\text { No (but multiple datasets / } \\
\text { non-trained) }\end{array}$ & $\begin{array}{l}\text { No, limited because of NYP/CUIMC } \\
\text { and Mayo notes. }\end{array}$ & [64] \\
\hline Lowe & 2009 & $\begin{array}{l}\text { Manual } \\
\text { retrospective } \\
\text { review }\end{array}$ & $\begin{array}{l}\text { Hold-out validation (train, } \\
\text { test) }\end{array}$ & No & $\begin{array}{l}\text { Yes, has the potential to index other } \\
\text { classes of clinical documents }\end{array}$ & [65] \\
\hline Luo & 2014 & $\begin{array}{l}\text { Existing EHR } \\
\text { data }\end{array}$ & 10-fold cross validation & No & $\begin{array}{l}\text { No, challenging, not currently working } \\
\text { on it }\end{array}$ & [66] \\
\hline Meystre & 2006 & $\begin{array}{l}\text { Manual } \\
\text { retrospective } \\
\text { review }\end{array}$ & Not used & No & Not listed & {$[67]$} \\
\hline Meystre & 2010 & $\begin{array}{l}\text { Existing } \\
\text { annotated } \\
\text { corpus }\end{array}$ & $\begin{array}{l}\text { Hold-out validation (train, } \\
\text { test) }\end{array}$ & No & Not listed & [68] \\
\hline Minard & 2011 & $\begin{array}{l}\text { Existing } \\
\text { annotated } \\
\text { corpus }\end{array}$ & $\begin{array}{l}\text { Hold-out validation (train, } \\
\text { test, development) }\end{array}$ & No & Not listed & [69] \\
\hline Mishra & 2019 & $\begin{array}{l}\text { Manual } \\
\text { annotations }\end{array}$ & Not used & No & Not listed & [70] \\
\hline Nguyen & 2018 & $\begin{array}{l}\text { Existing EHR } \\
\text { data }\end{array}$ & Not listed & No & Not listed & {$[71]$} \\
\hline Oellrich & 2015 & $\begin{array}{l}\text { Existing } \\
\text { annotated } \\
\text { corpus }\end{array}$ & External & Multiple datasets & Not listed & {$[72]$} \\
\hline Patrick & 2011 & $\begin{array}{l}\text { Existing } \\
\text { annotated }\end{array}$ & 10-fold cross validation & No & $\begin{array}{l}\text { Yes, adaptable to different requirements } \\
\text { in clinical information extraction and }\end{array}$ & [73] \\
\hline
\end{tabular}


Table 4 Included publications and their evaluation methodologies (Continued)

\begin{tabular}{|c|c|c|c|c|c|c|}
\hline Author & Year & Ref. std. & Validation & External & Generalizability $^{a}$ & Ref \\
\hline & & corpus & & & $\begin{array}{l}\text { classification by choosing relevant feature } \\
\text { sets }\end{array}$ & \\
\hline Pérez & 2018 & $\begin{array}{l}\text { Existing } \\
\text { annotated } \\
\text { corpus }\end{array}$ & $\begin{array}{l}\text { Hold-out validation (train, } \\
\text { test, development) }\end{array}$ & No & $\begin{array}{l}\text { Yes, extensible to different hospital-sections } \\
\text { and hospitals }\end{array}$ & {$[74]$} \\
\hline Reátegui & 2018 & $\begin{array}{l}\text { Existing } \\
\text { annotated } \\
\text { corpus }\end{array}$ & Not used & No & Not listed & [75] \\
\hline Roberts & 2011 & $\begin{array}{l}\text { Existing } \\
\text { annotated } \\
\text { corpus }\end{array}$ & $\begin{array}{l}\text { Hold-out validation (train, } \\
\text { test) }\end{array}$ & No & Not listed & [76] \\
\hline Rousseau & 2019 & $\begin{array}{l}\text { Manual } \\
\text { annotations }\end{array}$ & Not used & No & Not listed & [77] \\
\hline Savova & 2010 & $\begin{array}{l}\text { Manual } \\
\text { annotations }\end{array}$ & 10-fold cross validation & No & Yes, implemented in several applications & [78] \\
\hline Shivade & 2015 & $\begin{array}{l}\text { Manual } \\
\text { annotations }\end{array}$ & $\begin{array}{l}\text { Hold-out validation (train, } \\
\text { test) }\end{array}$ & No & Not listed & [11] \\
\hline Shoenbill & 2019 & $\begin{array}{l}\text { Manual } \\
\text { annotations }\end{array}$ & $\begin{array}{l}\text { Hold-out validation (train, } \\
\text { test) }\end{array}$ & No & $\begin{array}{l}\text { Yes, can allow further evaluation and } \\
\text { improvement in care delivery models } \\
\text { and treatment approaches to multiple } \\
\text { chronic illnesses }\end{array}$ & [79] \\
\hline Sohn & 2014 & $\begin{array}{l}\text { Manual } \\
\text { annotations }\end{array}$ & $\begin{array}{l}\text { Hold-out validation (train, } \\
\text { test, development) }\end{array}$ & No & $\begin{array}{l}\text { Yes, with adaptions: create flexible } \\
\text { mechanism for adaptation process }\end{array}$ & [80] \\
\hline Solti & 2008 & $\begin{array}{l}\text { Manual } \\
\text { annotations }\end{array}$ & $\begin{array}{l}\text { Hold-out validation (train, } \\
\text { test) }\end{array}$ & No & Not listed & [81] \\
\hline Soriano & 2019 & $\begin{array}{l}\text { Manual } \\
\text { annotations }\end{array}$ & Not listed & No & Not listed & [82] \\
\hline Soysal & 2018 & $\begin{array}{l}\text { Existing } \\
\text { annotated } \\
\text { corpus }\end{array}$ & $\begin{array}{l}\text { Hold-out validation (train, } \\
\text { test) }\end{array}$ & No & $\begin{array}{l}\text { Yes, can be used to quickly develop } \\
\text { customized clinical information extraction } \\
\text { pipelines }\end{array}$ & [83] \\
\hline Spasić & 2015 & $\begin{array}{l}\text { Manual } \\
\text { annotations }\end{array}$ & $\begin{array}{l}\text { Hold-out validation (train, } \\
\text { test) }\end{array}$ & No & Not listed & [84] \\
\hline Strauss & 2013 & $\begin{array}{l}\text { Manual } \\
\text { annotations }\end{array}$ & Not used & No & $\begin{array}{l}\text { Yes, can be shared between institutions } \\
\text { and used to support clinical + } \\
\text { epidemiological research }\end{array}$ & [85] \\
\hline Sung & 2018 & $\begin{array}{l}\text { Manual } \\
\text { annotations }\end{array}$ & Not listed & No & Not listed & [86] \\
\hline Tchechmedjiev & 2018 & $\begin{array}{l}\text { Existing } \\
\text { annotated } \\
\text { corpus }\end{array}$ & $\begin{array}{l}\text { Hold-out validation (train, } \\
\text { test, development) }\end{array}$ & No & Yes, but not universally & {$[87]$} \\
\hline Ternois & 2018 & $\begin{array}{l}\text { Existing EHR } \\
\text { data }\end{array}$ & $\begin{array}{l}5 \text {-fold cross validation }+ \\
\text { Hold-out validation (train, } \\
\text { test) }\end{array}$ & No & Not listed & [88] \\
\hline Travers & 2004 & $\begin{array}{l}\text { Manual } \\
\text { retrospective } \\
\text { review }\end{array}$ & Not used & No & Not listed & [89] \\
\hline Tulkens & 2019 & $\begin{array}{l}\text { Existing } \\
\text { annotated } \\
\text { corpus }\end{array}$ & $\begin{array}{l}\text { Hold-out validation (train, } \\
\text { test, development) }\end{array}$ & No & Not listed & [90] \\
\hline Usui & 2018 & $\begin{array}{l}\text { Manual } \\
\text { annotations }\end{array}$ & Not used & No & Not listed & [91] \\
\hline Valtchinov & 2019 & $\begin{array}{l}\text { Manual } \\
\text { annotations }\end{array}$ & Not used & No & No & [92] \\
\hline Wadia & 2018 & $\begin{array}{l}\text { Manual } \\
\text { annotations }\end{array}$ & Not used & No & Not listed & [93] \\
\hline Walker & 2019 & Manual & Hold-out validation & No & Yes, it can be incorporated in & [94] \\
\hline
\end{tabular}


Table 4 Included publications and their evaluation methodologies (Continued)

\begin{tabular}{|c|c|c|c|c|c|c|}
\hline Author & Year & Ref. std. & Validation & External & Generalizability $^{a}$ & Ref \\
\hline & & $\begin{array}{l}\text { retrospective } \\
\text { review }\end{array}$ & (development, test) & & institutional data warehouse & \\
\hline Xie & 2019 & $\begin{array}{l}\text { Existing } \\
\text { annotated } \\
\text { corpus }\end{array}$ & $\begin{array}{l}\text { Hold-out validation (train, } \\
\text { test, development) }\end{array}$ & No & Not listed & [95] \\
\hline Xu & 2011 & $\begin{array}{l}\text { Manual } \\
\text { annotations }\end{array}$ & $\begin{array}{l}\text { Hold-out validation (train, } \\
\text { test) }\end{array}$ & No & $\begin{array}{l}\text { Yes, generable approach to combine } \\
\text { information from heterogeneous data } \\
\text { sources in EHRs }\end{array}$ & [96] \\
\hline Yadav & 2013 & $\begin{array}{l}\text { Manual } \\
\text { annotations }\end{array}$ & Not used & No & $\begin{array}{l}\text { Yes, should be broadly applicate to } \\
\text { outcomes of clinical interest }\end{array}$ & [97] \\
\hline Yao & 2019 & $\begin{array}{l}\text { Existing } \\
\text { annotated } \\
\text { corpus }\end{array}$ & $\begin{array}{l}\text { Hold-out validation (train, } \\
\text { test) }\end{array}$ & No & Not listed & [98] \\
\hline Zeng & 2018 & $\begin{array}{l}\text { Manual } \\
\text { annotations }\end{array}$ & $\begin{array}{l}5 \text {-fold cross validation }+ \\
\text { Hold-out validation (train, } \\
\text { test) }\end{array}$ & No & Yes, potential to be replicated & [99] \\
\hline Zhang & 2013 & $\begin{array}{l}\text { Existing } \\
\text { annotated } \\
\text { corpus }\end{array}$ & External & $\begin{array}{l}\text { Two different sets with } \\
\text { same settings }\end{array}$ & $\begin{array}{l}\text { Yes, can be adapted to different } \\
\text { semantic categories and text genres }\end{array}$ & [100] \\
\hline Zhou & 2006 & $\begin{array}{l}\text { Manual } \\
\text { annotations }\end{array}$ & 5-fold cross validation & No & Not listed & [101] \\
\hline Zhou & 2011 & $\begin{array}{l}\text { Manual } \\
\text { retrospective } \\
\text { review }\end{array}$ & $\begin{array}{l}\text { Hold-out validation (train, } \\
\text { test) }\end{array}$ & No & Not listed & [102] \\
\hline Zhou & 2014 & $\begin{array}{l}\text { Manual } \\
\text { annotations }\end{array}$ & Not used & No & Not listed & [103] \\
\hline
\end{tabular}

${ }^{a}$ As reported by authors

- Not published in a peer-reviewed journal (except for ACL and ACM publications)

In the second phase, both reviewers excluded publications where the developed NLP algorithm was not evaluated by assessing the titles, abstracts, and, in case of uncertainty, the Method section of the publication. In the third phase, both reviewers independently evaluated the resulting full-text articles for relevance. The reviewers used Rayyan [27] in the first phase and Covidence [28] in the second and third phases to store the information about the articles and their inclusion. In all phases, both reviewers independently reviewed all publications. After each phase the reviewers discussed any disagreement until consensus was reached.

\section{Data extraction and categorization}

Both reviewers categorized the implementations of the found algorithms and noted their characteristics in a structured form in Covidence. The objectives of the included studies and their associated NLP tasks were categorized by way of induction. The results were compared and merged into one result set.

We collected the following characteristics of the studies, based on a combination of TRIPOD [21], STROBE
[22], RECORD [23], and STARD [24] statement elements (see Additional file 3): year, country, setting, objectives, evaluation methods, used NLP systems or algorithms, used terminology systems, size of datasets, performance measures, reference standard, language of the free-text data, validation methods, generalizability, operational use, and source code availability.

\section{List of recommendations}

Based on the findings of the systematic review and elements from the TRIPOD, STROBE, RECORD, and STARD statements, we formed a list of recommendations. The recommendations focus on the development and evaluation of NLP algorithms for mapping clinical text fragments onto ontology concepts and the reporting of evaluation results.

\section{Results}

The literature search generated a total of 2355 unique publications. After reviewing the titles and abstracts, we selected 256 publications for additional screening. Out of the 256 publications, we excluded 65 publications, as the described Natural Language Processing algorithms in those publications were not evaluated. The full text of the remaining 191 publications was assessed and 114 
Table 5 Characteristics of the included studies

\begin{tabular}{|c|c|c|}
\hline Description & n (\%) & References \\
\hline \multicolumn{3}{|l|}{ Main objective } \\
\hline Information extraction & $45(58 \%)$ & $\begin{array}{l}{[29,32-36,38,40-45,49,51,58-60,63-66,68-70,72,73,75,} \\
76,78-80,82,84-87,89,90,94,95,100,101,103,104]\end{array}$ \\
\hline Information enrichment & $9(12 \%)$ & {$[30,31,39,48,50,52,56,67,81]$} \\
\hline Classification & $8(10 \%)$ & {$[11,12,53,88,92,93,96,99]$} \\
\hline Software development and evaluation & $6(7.8 \%)$ & {$[37,46,47,61,83,102]$} \\
\hline Prediction & $4(5.2 \%)$ & {$[57,91,97,98]$} \\
\hline Information comparison & $2(2.6 \%)$ & {$[62,77]$} \\
\hline Computer-assisted coding & $2(2.6 \%)$ & {$[55,71]$} \\
\hline Text processing & $1(1.3 \%)$ & [74] \\
\hline \multicolumn{3}{|l|}{ Part of challenge } \\
\hline $\begin{array}{l}\text { i2b2 } \\
\text { (Informatics for Integrating Biology and the } \\
\text { Bedside) }\end{array}$ & $10(13 \%)$ & {$[11,44,47,58,68,69,73,76,78,83]$} \\
\hline Entire system & $8(10 \%)$ & {$[11,44,58,68,69,73,76,78]$} \\
\hline Parts of the system & $2(2.6 \%)$ & {$[47,83]$} \\
\hline SemEval (Semantic Evaluation) & $2(2.6 \%)$ & {$[41,83]$} \\
\hline Entire system & $1(1.3 \%)$ & [41] \\
\hline Parts of the system & $1(1.3 \%)$ & [83] \\
\hline $\begin{array}{l}\text { ShARe/CLEF } \\
\text { (Shared Annotated Resources/Conference and } \\
\text { Labs of the Evaluation Forum) }\end{array}$ & $1(1.3 \%)$ & [83] \\
\hline Parts of the system & $1(1.3 \%)$ & [83] \\
\hline \multicolumn{3}{|l|}{ Dataset: language } \\
\hline English & $60(78 \%)$ & $\begin{array}{l}{[11,12,29,30,32,35,37-39,41-47,49,53,55,56,58,} \\
60,62-73,75-81,83-86,89,90,92-104]\end{array}$ \\
\hline Spanish & $5(6.5 \%)$ & {$[31,36,52,74,82]$} \\
\hline French & $3(3.9 \%)$ & {$[51,87,88]$} \\
\hline German & $3(3.9 \%)$ & {$[33,34,61]$} \\
\hline Italian & $2(2.6 \%)$ & {$[40,43]$} \\
\hline Portuguese & $2(2.6 \%)$ & {$[48,50]$} \\
\hline Dutch & $1(1.3 \%)$ & {$[57]$} \\
\hline Japanese & $1(1.3 \%)$ & [91] \\
\hline Korean & $1(1.3 \%)$ & [59] \\
\hline \multicolumn{3}{|l|}{ Dataset: Origin } \\
\hline Data present in institute & $55(71 \%)$ & $\begin{array}{l}{[12,29,31,32,34-36,38-40,42,43,45,47,48,50-53,56,57,} \\
59-67,70,71,74,77-86,88,89,91-94,96,97,99,101-103]\end{array}$ \\
\hline Existing dataset & $25(33 \%)$ & $\begin{array}{l}{[11,30,33,35,37,41,44,46,49,55,58,64,68,69,72,73,75,} \\
76,83,87,90,95,98,100,104]\end{array}$ \\
\hline Included reference to dataset & $21(27 \%)$ & $\begin{array}{l}{[11,30,35,37,41,44,46,49,55,58,64,72,75,76,83,87,90,} \\
95,98,100,104]\end{array}$ \\
\hline \multicolumn{3}{|l|}{ Training of algorithm } \\
\hline Trained & $47(61 \%)$ & $\begin{array}{l}{[11,12,29,31,32,34,37,39,41,42,44,45,48-53,55-59,62,63,} \\
65,66,68,69,73,74,76,78-84,87,88,90,95,96,98,99,104]\end{array}$ \\
\hline Not listed & $3(3.9 \%)$ & {$[30,101,102]$} \\
\hline \multicolumn{3}{|l|}{ Development of algorithm } \\
\hline Use of development set & $16(21 \%)$ & {$[12,29,31,34,37,49,55,60,63,69,74,80,87,90,94,95]$} \\
\hline Not listed & $4(5.2 \%)$ & {$[30,82,83,101]$} \\
\hline
\end{tabular}


Table 5 Characteristics of the included studies (Continued)

\begin{tabular}{|c|c|c|}
\hline Description & n (\%) & References \\
\hline \multicolumn{3}{|l|}{ Used NLP system or algorithm } \\
\hline New NLP system or algorithm & $29(38 \%)$ & $\begin{array}{l}{[31,32,37,43,45,47-52,55,57,59,68,73,74,80,82,83,} \\
85,88,89,91,94,95,100-102]\end{array}$ \\
\hline $\begin{array}{l}\text { New NLP system or algorithm with existing } \\
\text { components }\end{array}$ & $25(33 \%)$ & $\begin{array}{l}{[12,29,34,39,41,42,44,46,58,60-63,66,67,69,71,75,} \\
76,78,84,87,90,98,99]\end{array}$ \\
\hline Existing NLP system or algorithm & $23(30 \%)$ & $\begin{array}{l}{[11,30,33,35,36,38,40,53,56,64,65,70,72,77,79,81,} \\
86,93,96,97,103,104]\end{array}$ \\
\hline \multicolumn{3}{|l|}{ Use in practice } \\
\hline $\begin{array}{l}\text { Plans to implement / still under development } \\
\text { and testing }\end{array}$ & $12(16 \%)$ & {$[31,33,51,56,62,66-68,82,91,96,101]$} \\
\hline Implemented in practice & $10(13 \%)$ & {$[34,42,43,46-48,78,83,87,102]$} \\
\hline \multicolumn{3}{|l|}{ Availability of code } \\
\hline Published algorithm or source code & $15(20 \%)$ & {$[31,45-47,60,78,80,82-85,87,90,97,98]$} \\
\hline Pseudocode in manuscript & $3(3.9 \%)$ & {$[43,56,62]$} \\
\hline Planning to publish algorithm or source code & $1(1.3 \%)$ & {$[32]$} \\
\hline Not applicable, used an existing system & $20(26 \%)$ & $\begin{array}{l}{[11,30,33,35,36,38,40,53,64,65,70,72,77,79,81} \\
86,93,96,103,104]\end{array}$ \\
\hline
\end{tabular}

publications did not meet our criteria, of which 3 publications in which the algorithm was not evaluated, resulting in 77 included articles describing 77 studies. Reference checking did not provide any additional publications. The PRISMA flow diagram is presented in Fig. 1.
The induction process resulted in eight categories and ten associated NLP tasks that describe the objectives of the papers: computer-assisted coding, information comparison, information enrichment, information extraction, prediction, software development and evaluation, and text

Table 6 Evaluation methods of the included studies

\begin{tabular}{|c|c|c|}
\hline Description & n (\%) & References \\
\hline \multicolumn{3}{|l|}{ Evaluation: Reference standard } \\
\hline Manual annotations & $40(52 \%)$ & $\begin{array}{l}{[11,12,32,34-36,38-40,42,43,45,47,48,51-53,56,59,60,62,64,70,77-82,} \\
84-86,91-93,96,97,99,101,103]\end{array}$ \\
\hline Existing annotated corpus & $24(31 \%)$ & {$[30,33,37,41,44,46,49,55,58,63,68,69,72-76,83,87,90,95,98,100,104]$} \\
\hline Existing EHR data & $7(9.1 \%)$ & {$[29,50,57,61,66,71,88]$} \\
\hline Manual retrospective review & $6(7.8 \%)$ & {$[31,65,67,89,94,102]$} \\
\hline \multicolumn{3}{|l|}{ Evaluation: Validation } \\
\hline Hold-out validation & $40(52 \%)$ & $\begin{array}{l}{[11,12,29,31,34,37,41,42,45,48-52,55,56,58-60,63,65,68,69,74,76,} \\
79-81,83,84,87,88,90,94-96,98,99,102,104]\end{array}$ \\
\hline Cross-validation & $12(16 \%)$ & {$[32,39,44,53,57,62,66,73,78,88,99,101]$} \\
\hline External validation & $9(12 \%)$ & {$[30,32,35,42,45,46,48,72,100]$} \\
\hline Solely external validation & $5(6.5 \%)$ & {$[30,35,46,72,100]$} \\
\hline In addition to another type of validation & $4(5.2 \%)$ & {$[32,42,45,48]$} \\
\hline Not performed or not listed & $22(29 \%)$ & {$[33,36,38,40,43,47,61,64,67,70,71,75,77,82,85,86,89,91-93,97,103]$} \\
\hline \multicolumn{3}{|l|}{ Generalizability } \\
\hline Claimed & $23(30 \%)$ & {$[30-32,35,38,45,49,51,58,59,65,73,74,78-80,83,85,87,94,96,97,100]$} \\
\hline Externally validated & $5(6.5 \%)$ & {$[30,32,35,45,100]$} \\
\hline \multicolumn{3}{|l|}{ Comparison } \\
\hline $\begin{array}{l}\text { Compared to other existing algorithms } \\
\text { or models }\end{array}$ & $24(31 \%)$ & {$[30,35,39,45-47,49,58,60,63,64,72,75,80,83,87,90,94,95,98-101,104]$} \\
\hline $\begin{array}{l}\text { Tested difference in outcomes for statistical } \\
\text { significance }\end{array}$ & $4(5.2 \%)$ & {$[35,39,60,63]$} \\
\hline
\end{tabular}


Table 7 Performance measures used in the included studies

\begin{tabular}{|c|c|c|c|}
\hline Description & Formula & n (\%) & References \\
\hline Confusion Matrix & $\begin{array}{l}\text { Lists the True Positives (TP), True Negatives (TN), False } \\
\text { Positives (FP), False Negatives (FN), and the Total (n) } \\
\text { amount in a } 2 \times 2 \text { contingency Table. } \\
\text { TP: Text annotated with ontology concept when } \\
\text { ontology concept is present in reference standard } \\
\text { TN: Text not annotated with ontology concept when } \\
\text { ontology concept is absent in reference standard } \\
\text { FP: Text annotated with ontology concept when } \\
\text { ontology concept is absent in reference standard } \\
\text { FN: Text not annotated with ontology concept when } \\
\text { ontology concept is present in reference standard }\end{array}$ & $12(16 \%)$ & {$[34,44,47,51,56,58,60,61,84,87,91,93]$} \\
\hline \multicolumn{4}{|l|}{ Performance measures } \\
\hline Recall & $\frac{T P}{F N+T P}$ & $68(88 \%)$ & $\begin{array}{l}{[11,12,29-31,33-53,56-58,60-64,66-73,75-88,} \\
90-94,96,99-104]\end{array}$ \\
\hline Precision & $\frac{T P}{F P+T P}$ & $66(86 \%)$ & $\begin{array}{l}{[11,12,29-31,33-36,38-51,53,56-58,60-73,75-88,} \\
90,91,93,94,96,99-104 \\
]\end{array}$ \\
\hline F-score & $2 \cdot \frac{\text { Precision-Recall }}{\text { Precision+Recall }}$ & $57(74 \%)$ & $\begin{array}{l}{[11,12,30,31,33-36,39-41,44,46-50,52,53,55} \\
57-63,66-73,75-80,82-84,86-88,90,91,95,96 \\
98-100,102-104]\end{array}$ \\
\hline Accuracy & $\frac{T P+T N}{n}$ & $11(14 \%)$ & {$[30,32,34,41,48,52,67,74,78,92,96]$} \\
\hline Specificity & $\frac{T N}{F P+T N}$ & $6(7.8 \%)$ & {$[29,34,85,92,93,96]$} \\
\hline$A \cup C$ & Not applicable & $5(6.5 \%)$ & {$[29,39,57,95,99]$} \\
\hline Kappa & $\frac{p_{o}-p_{e}}{1-p_{e}}=1-\frac{1-p_{o}}{1-p_{e}}$ & $3(3.9 \%)$ & {$[85,89,97]$} \\
\hline Processing time & Not applicable & $3(3.9 \%)$ & {$[32,47,83]$} \\
\hline Negative Predictive Value & $\frac{T N}{F N+T N}$ & $3(3.9 \%)$ & {$[29,85,93]$} \\
\hline False Positive Rate & $\frac{F P}{F P+T N}$ & $1(1.3 \%)$ & [34] \\
\hline False Negative Rate & $\frac{F N}{T P+F N}$ & $1(1.3 \%)$ & [34] \\
\hline Information entropy & $-\sum_{i=1}^{n} P_{i} \log \left(P_{i}\right)$ & $1(1.3 \%)$ & [64] \\
\hline Mean Reciprocal Rank & $\frac{1}{Q} \sum_{i=1}^{Q} \frac{1}{\operatorname{rank}_{i}}$ & $1(1.3 \%)$ & [74] \\
\hline Initial annotator agreement & Not applicable & $1(1.3 \%)$ & [79] \\
\hline Match/no match (\%) & Not applicable & $1(1.3 \%)$ & [89] \\
\hline Overgeneration & $\frac{F P}{T P+F P}$ & $1(1.3 \%)$ & [93] \\
\hline Undergeneration & $\frac{F N}{T P+F N}$ & $1(1.3 \%)$ & [68] \\
\hline Error & $\frac{F N+F P}{T P+F N+F P}$ & $1(1.3 \%)$ & [68] \\
\hline Fallout & $\frac{F P}{T N+F P}$ & $1(1.3 \%)$ & [68] \\
\hline Mean Standard Error & $\frac{1}{n} \sum_{i=1}^{n}\left(Y_{i}-\hat{Y}_{i}\right)^{2}$ & $1(1.3 \%)$ & [57] \\
\hline
\end{tabular}

processing. Our definitions of these NLP tasks and the associated categories are given in Table 1 and Table 2 .

Table 3 lists the included publications with their first author, year, title, and country. Table 4 lists the included publications with their evaluation methodologies. The non-induced data, including data regarding the sizes of the datasets used in the studies, can be found as supplementary material attached to this paper.

Table 5 summarizes the general characteristics of the included studies and Table 6 summarizes the evaluation methods used in these studies. In all 77 papers, we found twenty different performance measures (Table 7).

\section{Discussion}

In this systematic review, we reviewed the current state of NLP algorithms that map clinical text fragments onto ontology concepts with regard to their development and evaluation, in order to propose recommendations for future studies. 


\section{Main findings and recommendations}

We identified 256 studies that reported on the development of such algorithms, of which 68 did not evaluate the performance of the system. We included 77 studies. Many publications did not report their findings in a structured way, which made it challenging to extract all the data in a reliable manner. We discuss our findings and recommendations in the following five categories: Used NLP systems and algorithms, Used data, Evaluation and validation, Presentation of results, and Generalizability of results. A checklist for determining if the recommendations are followed in the reporting of an NLP study is added as supplementary material to this paper.

\section{Used NLP systems and algorithms}

A variety of NLP systems are used in the reviewed studies. Researchers use existing systems $(n=29$, $38 \%$ ), develop new systems with existing components $(n=25,33 \%)$, or develop a completely new system $(n=23,30 \%)$. Most studies, however, do not publish their (adapted) source code $(n=57,74 \%)$, and a description of the algorithm in the final publication is often not detailed enough to replicate it. To ensure reproducibility, implementation details, including details on data processing, and preferably the source code should be published, allowing other researchers to compare their implementations or to reproduce the results. Based on these findings, we formulated three recommendations (Table 8).

\section{Used data}

Most authors evaluate their algorithms with manual annotations $(n=40,52 \%)$ and use data present in their institutions $(n=55,71 \%)$. However, it is not clear what these datasets consist of. Most studies describe the data as 'reports', 'notes', or 'summaries', but do not list the contents or example rows from the dataset. It is, therefore, not clear what types of patients and what specific types of data are included, making

Table 8 Recommendation regarding the use of systems and algorithms

1. Describe the system or algorithm that is used or the system that is developed for the specific NLP task.

1. When an existing NLP system or algorithm is used, describe how it

is set up, how it is implemented in practice, and if and how the implementation differs from the original implementation.

2. When a new system is developed, describe the components and features used in the system, and preferably include a flow chart that explains how these elements work together.

2. Include the source code of the developed algorithm as

supplementary material to the publication or upload the source code to a repository such as GitHub.

3. Specify which ontologies are used in the encoding task, including the version of the ontology.

1. If a new ontology is developed for the encoding task, report on

the development and content of the ontology and rationale for the development of a new ontology instead of the use of an existing one. The MIRO guidelines could be used to structure the report [105].
Table 9 Recommendation regarding the use of data

1. To ensure that new algorithms can be compared against your system, aim to publish the used training, development, and validation data in a data repository.

1. In case the data cannot be published, determine if the data can be accessed on request or can be used in a federated learning approach (i.e., a learning process in which the data owners collaboratively train a model in which process any data owner does not expose the data to others [107]).

2. In case a reference standard is used, include information about the origin of the data (external dataset, subset of the dataset) and the characteristics of the data in the dataset. If possible, reference the dataset using a DOI or URL.

3. If an external dataset is used, give a short description of the data present in the dataset and reference the source of the dataset.

the study hard to reproduce. Finally, we found a wide range of dataset sizes and formats. The training datasets, for example, ranged from 10 clinical notes to 636.439 discharge reports. The use of small datasets can result in an overfitted algorithm that either performs well on the dataset, but not on an external dataset, or performs poorly, for the algorithm was only trained on a specific type of data. More difficult recognition tasks require more data, and therefore sample size planning is recommended [106]. To improve the description and availability of datasets used in NLP studies, we formulated three recommendations (Table 9).

\section{Evaluation and validation}

Evaluation of the algorithm determines its performance on the dataset, and validation determines if the algorithm is not overfitted on that dataset and thus if the algorithm might work on other datasets as well. Over one-fourth of the studies $(n=68,27 \%)$ that we identified did not evaluate their algorithms. In addition, 22 included studies (29\%) did not validate the developed algorithm. A statement claiming that an algorithm can be used in clinical practice can be questioned if the algorithm has not been evaluated and validated. Across all studies, 20 performance measures were used. To harmonize evaluation and validation efforts, we formulated three recommendations (Table 10).

Table 10 Recommendation regarding the evaluation and validation of Natural Language Processing algorithms

1. Perform an evaluation using generic (i.e., precision, recall, and F-score) performance measures and appropriate aspects of evaluation including discrimination, calibration, and preferably accuracies of predictions (e.g., AUC, calibration graphs, and the Brier score).

1. Include a motivation for the choice of measures, with references to existing literature where appropriate (e.g., Sokolova and Lapalme's analysis of performance measures [108]).

2. Perform an error analysis and discuss the errors in the Discussion section of the paper. Include possible changes to the algorithm that could improve its performance for these specific errors.

3. When using a non-probabilistic NLP method: determine the cut-off value (a priori) for a 'good' test result before evaluating the algorithm. Elaborate why this cut-off value is chosen. 


\section{Presentation of results}

Authors report the evaluation results in various formats. Only twelve articles (16\%) included a confusion matrix which helps the reader understand the results and their impact. Not including the true positives, true negatives, false positives, and false negatives in the Results section of the publication, could lead to misinterpretation of the results of the publication's readers. For example, a high Fscore in an evaluation study does not directly mean that the algorithm performs well. There is also a possibility that out of 100 included cases in the study, there was only one true positive case, and 99 true negative cases, indicating that the author should have used a different dataset. Results should be clearly presented to the user, preferably in a table, as results only described in the text do not provide a proper overview of the evaluation outcomes (Table 11). This also helps the reader interpret results, as opposed to having to scan a free text paragraph. Most publications did not perform an error analysis, while this will help to understand the limitations of the algorithm and implies topics for future research.

\section{Generalizability of results}

$88 \%$ of the studies did not perform external validation $(n=68)$. Of the studies that claimed that their algorithm was generalizable, only $22 \%(n=5)$ assessed this claim through external validation. However, one cannot claim generalizability without testing for it. Moreover, in 19\% $(n=3)$ of the cases where external datasets were used, the datasets were not referenced and only listed in the text of the article, making it harder to find the used data and reproduce the results. Algorithm performance should be compared to that of other state-of-the-art algorithms, as this helps the reader decide whether the new algorithm could be considered useful for clinical practice. However, only 24 studies (31\%) made this comparison, and four of those studies (17\%) tested the performance difference for statistical significance. We also found that the authors' descriptions of generalizability are rather ambiguous and unclear. We formulated five recommendations regarding the generalizability of results (Table 12).

Table 11 Recommendation regarding the presentation of results

1. Report the outcomes of the evaluation in a clear manner, preferably in a table accompanied by a textual description of the outcomes.

1. Aim to include a confusion matrix in the reporting of the outcomes.

2. Use figures if they contribute to the making the results more readable and understandable for the reader. If a figure is used, make sure that the data is also available in the text or in a table.
Table 12 Recommendation regarding the generalizability of results

1. Compare the results of the evaluated algorithm with other algorithms by using the same dataset as reported in the publication of the other algorithm or by processing the same dataset with another algorithm available through the literature. Report the outcomes of both experiments and test for statistical significance.

2. Describe in what setting the research is performed. Include if the research is part of a challenge (e.g., i2b2 challenge), or that the research is carried out in a specific institute or department.

3. Before claiming generalizability, perform external validation by testing the algorithm on a different, external dataset from other research projects or other publicly available datasets. Aim to use a dataset with a different case mix, different individuals, and different types of text. 4. Determine and describe if there are potential sources of bias in data selection, data use by the NLP algorithm or system, and evaluation. 5. When claiming generalizability, clearly describe the conditions under which the algorithm can be used in a different setting. Describe for which population, domain, and type and language of data the algorithm can be used.

\section{Strengths}

Our study has three main strengths: First, to our knowledge, this is the first systematic review that focuses on the evaluation of NLP algorithms in medicine. Second, we used a large number of databases for our search, resulting in publications from many different sources, such as medical journals and computer science conferences. Third, we used existing statements and guidelines and harmonized them to induce our findings and used these findings to propose a list of recommendations.

\section{Limitations}

Several limitations of our study should be noted as well. First, we only focused on algorithms that evaluated the outcomes of the developed algorithms. Second, the majority of the studies found by our literature search used NLP methods that are not considered to be state of the art. We found that only a small part of the included studies was using state-ofthe-art NLP methods, such as word and graph embeddings. This indicates that these methods are not broadly applied yet for algorithms that map clinical text to ontology concepts in medicine and that future research into these methods is needed. Lastly, we did not focus on the outcomes of the evaluation, nor did we exclude publications that were of low methodological quality. However, we feel that NLP publications are too heterogeneous to compare and that including all types of evaluations, including those of lesser quality, gives a good overview of the state of the art.

\section{Conclusion}

In this study, we found many heterogeneous approaches to the development and evaluation of NLP 
algorithms that map clinical text fragments to ontology concepts and the reporting of the evaluation results. Over one-fourth of the publications that report on the use of such NLP algorithms did not evaluate the developed or implemented algorithm. In addition, over one-fourth of the included studies did not perform a validation and nearly nine out of ten studies did not perform external validation. Of the studies that claimed that their algorithm was generalizable, only one-fifth tested this by external validation. Based on the assessment of the approaches and findings from the literature, we developed a list of sixteen recommendations for future studies. We believe that our recommendations, along with the use of a generic reporting standard, such as TRIPOD, STROBE, RECORD, or STARD, will increase the reproducibility and reusability of future studies and algorithms.

\section{Supplementary Information}

Supplementary information accompanies this paper at https://doi.org/10. 1186/s13326-020-00231-z.

\section{Additional file 1. \\ Additional file 2. \\ Additional file 3 .}

\section{Abbreviations}

ACL: Association for Computational Linguistics; ACM: Association for Computing Machinery; EHR: Electronic Health Record; FN: False Negatives; FP: False Positives; HPO: Human Phenotype Ontology; NLP: Natural Language Processing; PRISMA: Preferred Reporting Items for Systematic reviews and Meta-Analyses; TN: True Negatives; TP: True Positives

\section{Acknowledgements}

Not applicable.

\section{Authors' contributions}

Study conception and design: MK, RC, DA, and AA. Acquisition of data: FP and MK. Analysis and interpretation of data: FP and MK. Drafting of manuscript: MK. Critical revision: RC, DA, and AA. All authors read and approved the final manuscript.

\section{Funding}

This work was supported by Castor EDC and the European Regional Development Fund (ERDF).

\section{Availability of data and materials}

All data generated or analysed during the study are included in this published article and its supplementary information files.

\section{Ethics approval and consent to participate}

Not applicable.

\section{Consent for publication}

Not applicable.

\section{Competing interests}

The authors declare that they have no competing interests.
Received: 17 July 2020 Accepted: 3 November 2020

Published online: 16 November 2020

\section{References}

1. Ford E, Nicholson A, Koeling R, Tate AR, Carroll J, Axelrod L, et al. Optimising the use of electronic health records to estimate the incidence of rheumatoid arthritis in primary care: what information is hidden in free text? BMC Med Res Methodol. 2013;13.

2. Rosenbloom ST, Denny JC, XU H, Lorenzi N, Stead WW, Johnson KB. Data from clinical notes: a perspective on the tension between structure and flexible documentation. J Am Med Informatics Assoc. 2011;18:181-6.

3. Coorevits P, Sundgren M, Klein GO, Bahr A, Claerhout B, Daniel C, et al. Electronic health records: new opportunities for clinical research. J Intern Med. 2013;274:547-60

4. Danciu I, Cowan JD, Basford M, Wang X, Saip A, Osgood S, et al. Secondary use of clinical data: the Vanderbilt approach. J Biomed Inform. 2014;52:28-35.

5. Price SJ, Stapley SA, Shephard E, Barraclough K, Hamilton WT. Is omission of free text records a possible source of data loss and bias in clinical practice research Datalink studies? A case-control study. BMJ Open. 2016;6.

6. Gruber TR. A translation approach to portable ontology specifications. Knowl Acquis. 1993:5:199-220.

7. SNOMED International. SNOMED CT http://www.snomed.org/snomed-ct/ five-step-briefing. Accessed 29 Jun 2020.

8. Köhler S, Carmody L, Vasilevsky N, Jacobsen JOB, Danis D, Gourdine JP, et al. Expansion of the human phenotype ontology (HPO) knowledge base and resources. Nucleic Acids Res. 2019;47:D1018-27.

9. Krasowski M, Schriever A, Mathur G, Blau J, Stauffer S, Ford B. Use of a data warehouse at an academic medical center for clinical pathology quality improvement, education, and research. J Pathol Inform. 2015;6:45.

10. Wu H, Toti G, Morley Kl, Ibrahim ZM, Folarin A, Jackson R, et al. SemEHR: a general-purpose semantic search system to surface semantic data from clinical notes for tailored care, trial recruitment, and clinical research. J Am Med Inf Assoc. 2018;25:530-7.

11. Shivade C, Malewadkar P, Fosler-Lussier E, Lai AM. Comparison of UMLS terminologies to identify risk of heart disease using clinical notes. J Biomed Inform. 2015:58:5103-10.

12. Lingren $T$, Thaker $V$, Brady C, Namjou B, Kennebeck S, Bickel J, et al. Developing an algorithm to detect early childhood obesity in two tertiary pediatric medical centers. Appl Clin Inform. 2016;7(3):693-706.

13. Ni Y, Kennebeck S, Dexheimer JW, McAneney $C M$, Tang $H$, Lingren $T$, et al. Automated clinical trial eligibility prescreening: increasing the efficiency of patient identification for clinical trials in the emergency department. J Am Med Informatics Assoc. 2015;22:166-78.

14. Sun H, Depraetere K, De Roo J, Mels G, De Vloed B, Twagirumukiza M, et al. Semantic processing of EHR data for clinical research. J Biomed Inform. 2015;58:247-59.

15. Kreimeyer K, Foster M, Pandey A, Arya N, Halford G, Jones SF, et al. Natural language processing systems for capturing and standardizing unstructured clinical information: a systematic review. J Biomed Inf. 2017:73:14-29.

16. Gonzalez-Hernandez G, Sarker A, O'Connor K, Savova G. Capturing the Patient's perspective: a review of advances in natural language processing of health-related text. Yearb Med Inf. 2017;26:214-27.

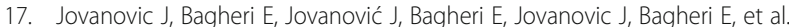
Semantic annotation in biomedicine: the current landscape. J Biomed Semant. 2017:8:44.

18. UK EQUATOR Centre. The EQUATOR Network. https://www.equator-network org/. Accessed 29 Jun 2020.

19. Ford E, Carroll JA, Smith HE, Scott D, Cassell JA. Extracting information from the text of electronic medical records to improve case detection: a systematic review. J Am Med Informatics Assoc. 2016;23:1007-15.

20. Vuokko R, Makela-Bengs P, Hypponen H, Lindqvist M, Doupi P, MäkeläBengs $P$, et al. Impacts of structuring the electronic health record: results of a systematic literature review from the perspective of secondary use of patient data. Int J Med Inform. 2017;97:293-303.

21. Collins GS, Reitsma JB, Altman DG, Moons KGM, TRIPOD Group. Transparent reporting of a multivariable prediction model for individual prognosis or diagnosis (TRIPOD): the TRIPOD statement. TRIPOD Group Circ. 2015;131:211-9.

22. von Elm E, Altman DG, Egger M, Pocock SJ, Gøtzsche PC, Vandenbroucke $J P$. The strengthening the reporting of observational studies in 
epidemiology (STROBE) statement: guidelines for reporting observational studies. J Clin Epidemiol. 2008;61:344-9.

23. Benchimol El, Smeeth L, Guttmann A, Harron K, Moher D, Peteresen I et al. The REporting of studies Conducted using Observational Routinelycollected health Data (RECORD) Statement. PLoS Med. 2015;12:1-22.

24. Bossuyt PM, Reitsma JB, Bruns DE, Gatsonis CA, Glasziou PP, Irwig L, et al. STARD 2015: an updated list of essential items for reporting diagnostic accuracy studies. BMJ. 2015;351:h5527.

25. Moher D, Liberati A, Tetzlaff J, Altman DG, Altman D, Antes G et al. Preferred reporting items for systematic reviews and meta-analyses: The PRISMA statement. PLoS Med. 2009;6:1-6.

26. The EndNote Team. EndNote. Philadelphia: Clarivate; 2013.

27. Ouzzani M, Hammady H, Fedorowicz Z, Elmagarmid A. Rayyan — a web and mobile app for systematic reviews. Syst Rev. 2016;5:210.

28. Veritas Health Innovation. Covidence systematic review software. Melbourne: Veritas Health Innovation; 2020.

29. Afshar M, Dligach D, Sharma B, Cai X, Boyda J, Birch S, et al. Development and application of a high throughput natural language processing architecture to convert all clinical documents in a clinical data warehouse into standardized medical vocabularies. J Am Med Inform Assoc. 2019;26:1364-9.

30. Alnazzawi $\mathrm{N}$, Thompson P, Ananiadou S. Mapping Phenotypic Information in Heterogeneous Textual Sources to a Domain-Specific Terminological Resource. PLoS One. 2016;11(9):e0162287.

31. Atutxa A, Perez A, Casillas A. Machine Learning Approaches on Diagnostic Term Encoding with the ICD for Clinical Documentation. IEEE J Biomed Heal Informatics. 2018;22(4):1323-9.

32. Barrett $\mathrm{N}$, Weber-Jahnke $\mathrm{JH}$, Thai V. Engineering natural language processing solutions for structured information from clinical text: extracting sentinel events from palliative care consult letters. Stud Health Technol Inform. 2013;192:594-8.

33. Becker M, Bockmann B. Extraction of UMLS(R) Concepts Using Apache cTAKES for German Language. Stud Health Technol Inform. 2016;223:PG-71-6.

34. Becker M, Kasper S, Böckmann B, Jöckel K-H, Virchow I. Natural language processing of German clinical colorectal cancer notes for guideline-based treatment evaluation. Int J Med Inform. 2019;127:141-6.

35. Bejan CA, Wei WQ, Denny JC. Assessing the role of a medication-indication resource in the treatment relation extraction from clinical text. J Am Med Informatics Assoc. 2015;22:e162-76.

36. Castro E, Iglesias A, Martínez P, Castaño L. Automatic Identification of Biomedical Concepts in Spanish-language Unstructured Clinical Texts. German Research Cent for Artificial, Intelligence - DFKI GmbH, Kaiserslautern, Germany Seattle, WA, USA: ACM; 2010. p. 751-7.

37. Catling F, Spithourakis GP, Riedel S. Towards automated clinical coding. Int J Med Inform. 2018;120:50-61.

38. Chapman WW, Fiszman M, Dowling JN, Chapman BE, Rindflesch TC. Identifying respiratory findings in emergency department reports for biosurveillance using MetaMap. Medinfo. 2004;11:487-91.

39. Chen J, Zheng J, Yu H. Finding Important Terms for Patients in Their Electronic Health Records: A Learning-to-Rank Approach Using Expert Annotations. JMIR Med informatics. 2016;4(4):e40.

40. Chiaramello E, Pinciroli F, Bonalumi A, Caroli A, Tognola G. Use of "off-the-shelf" information extraction algorithms in clinical informatics: A feasibility study of MetaMap annotation of Italian medical notes. J Biomed Inform. 2016;63:22-32.

41. Chodey KP, Hu G. Clinical text analysis using machine learning methods. In: 2016 IEEE/ACIS 15th International Conference on Computer and Information Science (ICIS); 2016. p. 1-6.

42. Chung J, Murphy S. Concept-value pair extraction from semi-structured clinical narrative: a case study using echocardiogram reports. AMIA Annu Symp Proc. 2005:131-5.

43. Combi C, Zorzi M, Pozzani G, Moretti U, Arzenton E. From narrative descriptions to MedDRA: automagically encoding adverse drug reactions. J Biomed Inform. 2018:84:184-99.

44. de Bruijn B, Cherry C, Kiritchenko S, Martin J, Zhu X. Machine-learned solutions for three stages of clinical information extraction: The state of the art at i2b2 2010. J Am Med Informatics Assoc. 2011;18(5):557-62.

45. Deisseroth CA, Birgmeier J, Bodle EE, Kohler JN, Matalon DR, Nazarenko Y, et al. ClinPhen extracts and prioritizes patient phenotypes directly from medical records to expedite genetic disease diagnosis. Genet Med. 2019;21:1585-93.

46. Demner-Fushman D, Rogers WJ, Aronson AR. MetaMap Lite: An evaluation of a new Java implementation of MetaMap. J Am Med Informatics Assoc. 2017;24(4):841-4.
47. Divita G, Zeng QT, Gundlapalli AV, Duvall S, Nebeker J, Samore MH. Sophia: A Expedient UMLS Concept Extraction Annotator. AMIA Annu Symp Proc. 2014;2014:467-76.

48. Duarte F, Martins B, Pinto CS, Silva MJ. Deep neural models for ICD-10 coding of death certificates and autopsy reports in free-text. J Biomed Inform. 2018:80:64-77.

49. Falis M, Pajak M, Lisowska A, Schrempf P, Deckers L, Mikhael S, et al. Ontological attention ensembles for capturing semantic concepts in ICD code prediction from clinical text; 2019. p. 168-77.

50. Ferrão JC, Janela F, Oliveira MD, HMG M. Using Structured EHR Data and SVM to Support ICD-9-CM Coding. In: 2013 IEEE International Conference on Healthcare Informatics; 2013. p. 511-6.

51. Gerbier S, Yarovaya O, Gicquel Q, Millet A-L, Smaldore V, Pagliaroli V, et al. Evaluation of natural language processing from emergency department computerized medical records for intra-hospital syndromic surveillance. BMC Med Inform Decis Mak. 2011;11:50.

52. Goicoechea Salazar JA, Nieto García MA, Laguna Téllez A, Canto Casasola VD, Rodríguez Herrera J, Murillo CF. Development of an automated coding system to retrieve and analyze diagnostic information stored in hospital emergency department records. Emergencias. 2013;25(6):430-6.

53. Hamid H, Fodeh SJ, Lizama AG, Czlapinski R, Pugh MJ, LaFrance WC Jr, et al. Validating a natural language processing tool to exclude psychogenic nonepileptic seizures in electronic medical record-based epilepsy research. Epilepsy Behav. 2013;29:578-80.

54. Hassanzadeh H, Kholghi M, Nguyen A, Chu K. Clinical document classification using labeled and unlabeled data across hospitals. AMIA Annu Symp proceedings AMIA Symp. 2018;2018:545-54.

55. Helwe C, Elbassuoni S, Geha M, Hitti E, Makhlouf OC. CCS Coding of Discharge Diagnoses via Deep Neural Networks. German Research Cent for Artificial, Intelligence - DFKI GmbH, Kaiserslautern, Germany Seattle, WA, USA: ACM; 2017. p. 175-9.

56. Hersh W, Mailhot M, Arnott-Smith C, Lowe H. Selective automated indexing of findings and diagnoses in radiology reports. J Biomed Inform. 2001;34(4):262-73.

57. Hoogendoorn M, Szolovits P, Moons LMG, Numans ME. Utilizing uncoded consultation notes from electronic medical records for predictive modeling of colorectal cancer. Artif Intell Med. 2015;69:53-61.

58. Jindal P, Roth D. Extraction of events and temporal expressions from clinical narratives. J Biomed Inform. 2013:46:S13-9.

59. Kang BY, Kim DW, Kim HG. Two-phase chief complaint mapping to the UMLS metathesaurus in Korean Electronic Medical Records. IEEE Trans Inf Technol Biomed. 2009;13(1):78-86.

60. Kersloot MGMG, Lau F, Abu-Hanna A, Arts DLDL, Cornet R. Automated SNOMED CT concept and attribute relationship detection through a webbased implementation of CTAKES. J Biomed Semantics. 2019;10:14.

61. König M, Sander A, Demuth I, Diekmann D, Steinhagen-Thiessen E. Knowledge-based best of breed approach for automated detection of clinical events based on German free text digital hospital discharge letters. PLoS One. 2019;14:e0224916.

62. Li Q, Spooner SA, Kaiser M, Lingren N, Robbins J, Lingren T, et al. An end-toend hybrid algorithm for automated medication discrepancy detection. BMC Med Inform Decis Mak. 2015;15:37.

63. Li F, Jin Y, Liu W, Rawat BPS, Cai P, Yu H. Fine-tuning bidirectional encoder representations from transformers (BERT)-based models on large-scale electronic health record notes: an empirical study. JMIR Med informatics. 2019;7:e14830

64. Liu C, Ta CN, Rogers JR, Li Z, Lee J, Butler AM, et al. Ensembles of natural language processing systems for portable phenotyping solutions. J Biomed Inform. 2019;100:103318.

65. Lowe HJ, Huang Y, Regula DP. Using a statistical natural language Parser augmented with the UMLS specialist lexicon to assign SNOMED CT codes to anatomic sites and pathologic diagnoses in full text pathology reports. AMIA Annu Symp Proc. 2009;2009:386-90.

66. Luo Y, Sohani AR, Hochberg EP, Szolovits P. Automatic lymphoma classification with sentence subgraph mining from pathology reports. J Am Med Informatics Assoc. 2014;21(5):824-32.

67. Meystre S, Haug PJ. Natural language processing to extract medical problems from electronic clinical documents: performance evaluation. J Biomed Inform. 2006;39(6):589-99.

68. Meystre SM, Thibault J, Shen S, Hurdle JF, South BR. Automatically detecting medications and the reason for their prescription in clinical narrative text documents. Stud Health Technol Inform. 2010;160(Pt 2):944-8. 
69. Minard AL, Ligozat AL, Abacha AB, Bernhard D, Cartoni B, Deléger $L$, et al. Hybrid methods for improving information access in clinical documents: Concept, assertion, and relation identification. J Am Med Informatics Assoc. 2011;18(5):588-93.

70. Mishra R, Burke A, Gitman B, Verma P, Engelstad M, Haendel MA, et al. Datadriven method to enhance craniofacial and oral phenotype vocabularies. J Am Dent Assoc. 2019;150:933-9 e2.

71. Nguyen AN, Truran D, Kemp M, Koopman B, Conlan D, O'Dwyer J, et al. Computer-assisted diagnostic coding: effectiveness of an NLP-based approach using SNOMED CT to ICD-10 mappings. AMIA . Annu Symp proceedings AMIA Symp. 2018;2018:807-16.

72. Oellrich A, Collier N, Smedley D, Groza T. Generation of silver standard concept annotations from biomedical texts with special relevance to phenotypes. PLoS One. 2015;10(1):e0116040.

73. Patrick JD, Nguyen DHM, Wang Y, Li M. A knowledge discovery and reuse pipeline for information extraction in clinical notes. J Am Med Informatics Assoc. 2011;18(5):574-9.

74. Pérez A, Atutxa A, Casillas A, Gojenola K, Sellart Á. Inferred joint multigram models for medical term normalization according to ICD. Int J Med Inform 2018;110:111-7.

75. Reátegui R, Ratté S. Comparison of MetaMap and cTAKES for entity extraction in clinical notes. BMC Med Inform Decis Mak. 2018;18(Suppl 3):74.

76. Roberts K, Harabagiu SM. A flexible framework for deriving assertions from electronic medical records. J Am Med Informatics Assoc. 2011;18(5):568-73.

77. Rousseau JF, Ip IK, Raja AS, Valtchinov VI, Cochon L, Schuur JD, et al. Can automated retrieval of data from emergency department physician notes enhance the imaging order entry process? Appl Clin Inform. 2019;10:189-98.

78. Savova GK, Masanz JJ, Ogren PV, Zheng J, Sohn S, Kipper-Schuler KC, et al. Mayo clinical text analysis and knowledge extraction system (CTAKES): architecture, component evaluation and applications. J Am Med Informatics Assoc. 2010;17:507-13.

79. Shoenbill K, Song Y, Gress L, Johnson H, Smith M, Mendonca EA. Natural language processing of lifestyle modification documentation. Health Informatics J. 2019:1460458218824742.

80. Sohn S, Clark C, Halgrim SR, Murphy SP, Chute CG, Liu H. MedXN: An open source medication extraction and normalization tool for clinical text. J Am Med Informatics Assoc. 2014;21(5):858-65.

81. Solti I, Aaronson B, Fletcher G, Solti M, Gennari JH, Cooper M, et al. Building an automated problem list based on natural language processing: lessons learned in the early phase of development. AMIA Annu Symp Proc. 2008; 2008:687-91.

82. Soriano IM, Peña JLC, Breis JTF, Román IS, Barriuso AA, Baraza DG. Snomed2Vec: Representation of SNOMED CT Terms with Word2Vec. In: 2019 IEEE 32nd International Symposium on Computer-Based Medical Systems (CBMS); 2019. p. 678-83

83. Soysal $E$, Wang J, Jiang M, Wu Y, Pakhomov S, Liu H, et al. CLAMP - a toolkit for efficiently building customized clinical natural language processing pipelines. J Am Med Informatics Assoc. 2018;25(3):331-6.

84. Spasić I, Zhao B, Jones CB, Button K. KneeTex: An ontology-driven system for information extraction from MRI reports. J Biomed Semantics. 2015;6:34.

85. Strauss JA, Chao CR, Kwan ML, Ahmed SA, Schottinger JE, Quinn VP. Identifying primary and recurrent cancers using a SAS-based natural language processing algorithm. J Am Med Informatics Assoc. 2013;20(2):349-55.

86. Sung SF, Chen K, Wu DP, Hung LC, Su YH, Hu YH. Applying natural language processing techniques to develop a task-specific EMR interface for timely stroke thrombolysis: A feasibility study. Int J Med Inform. 2018;112:149-57.

87. Tchechmedjiev A, Abdaoui A, Emonet V, Zevio S, Jonquet C. SIFR annotator: ontology-based semantic annotation of French biomedical text and clinical notes. BMC Bioinformatics. 2018;19:405.

88. Ternois I, Escudie J-B, Benamouzig R, Duclos C. Development of an automatic coding system for digestive endoscopies. Stud Health Technol Inform. 2018;255:107-11.

89. Travers DA, Haas SW. Evaluation of Emergency Medical Text Processor, a system for cleaning chief complaint text data. Acad Emerg Med. 2004. 11(11):1170-6

90. Tulkens S, Šuster S, Daelemans W. Unsupervised concept extraction from clinical text through semantic composition. J Biomed Inform. 2019;91:103120.

91. Usui M, Aramaki E, Iwao T, Wakamiya S, Sakamoto T, Mochizuki M. Extraction and standardization of patient complaints from electronic medication histories for Pharmacovigilance: natural language processing analysis in Japanese. JMIR Med informatics. 2018;6:e11021.
92. Valtchinov VI, Lacson R, Wang A, Khorasani R. Comparing Artificial Intelligence Approaches to Retrieve Clinical Reports Documenting Implantable Devices Posing MRI Safety Risks. J Am Coll Radiol. 2019;S1546-1440(19):30862.

93. Wadia R, Akgun K, Brandt C, Fenton BT, Levin W, Marple AH, et al. Comparison of natural language processing and manual coding for the identification of cross-sectional imaging reports suspicious for lung Cancer. JCO Clin cancer informatics. 2018;2:1-7.

94. Walker G, Soysal E, Xu H. Development of a natural language processing tool to extract radiation treatment sites. Cureus. 2019;11:e6010.

95. Xie X, Xiong Y, Yu PS, Zhu Y. EHR Coding with Multi-scale Feature Attention and Structured Knowledge Graph Propagation. ACM; 2019. p. 649-58.

96. Xu H, Fu Z, Shah A, Chen Y, Peterson NB, Chen Q, et al. Extracting and integrating data from entire electronic health records for detecting colorectal cancer cases. AMIA Annu Symp Proc. 2011;2011:1564-72.

97. Yadav K, Sarioglu E, Smith M, Choi HA. Automated outcome classification of emergency department computed tomography imaging reports. Acad Emerg Med. 2013;20(8PG):848-54

98. Yao L, Mao C, Luo Y. Clinical text classification with rule-based features and knowledge-guided convolutional neural networks. BMC Med Inform Decis Mak. 2019;19(Suppl 3):71.

99. Zeng Z, Espino S, Roy A, Li X, Khan SA, Clare SE, et al. Using natural language processing and machine learning to identify breast cancer local recurrence. BMC Bioinformatics. 2018;19(Suppl 17):498.

100. Zhang S, Elhadad N. Unsupervised biomedical named entity recognition: Experiments with clinical and biological texts. J Biomed Inform. 2013;46(6 PG):1088-98.

101. Zhou X, Han H, Chankai I, Prestrud A, Brooks A. Approaches to Text Mining for Clinical Medical Records. In: German Research Cent for Artificial, Intelligence DFKI GmbH, Kaiserslautern, Germany Seattle, WA, USA: ACM; 2006. p. 235-9.

102. Zhou L, Plasek JM, Mahoney LM, Karipineni N, Chang F, Yan X, et al. Using Medical Text Extraction, Reasoning and Mapping System (MTERMS) to process medication information in outpatient clinical notes. AMIA Annu Symp Proc. 2011;2011:1639-48.

103. Zhou L, Lu Y, Vitale CJ, Mar PL, Chang F, Dhopeshwarkar N, et al. Representation of information about family relatives as structured data in electronic health records. Appl Clin Inform. 2014;5:349-67.

104. Hassanzadeh H, Nguyen A, Koopman B. Evaluation of Medical Concept Annotation Systems on Clinical Records; 2016. p. 15-24.

105. Matentzoglu N, Malone J, Mungall C, Stevens R. MIRO: guidelines for minimum information for the reporting of an ontology. J Biomed Semantics. 2018;9:1-13.

106. Beleites C, Neugebauer U, Bocklitz T, Krafft C, Popp J. Sample size planning for classification models. Anal Chim Acta. 2013;760:25-33.

107. Yang Q, Liu Y, Chen T, Tong Y. Federated machine learning: concept and applications. ACM Trans Intell Syst Technol. 2019;10:1-19.

108. Sokolova M, Lapalme G. A systematic analysis of performance measures for classification tasks. Inf Process Manag. 2009;45:427-37.

\section{Publisher's Note}

Springer Nature remains neutral with regard to jurisdictional claims in published maps and institutional affiliations.

Ready to submit your research? Choose BMC and benefit from:

- fast, convenient online submission

- thorough peer review by experienced researchers in your field

- rapid publication on acceptance

- support for research data, including large and complex data types

- gold Open Access which fosters wider collaboration and increased citations

- maximum visibility for your research: over $100 \mathrm{M}$ website views per year

At $\mathrm{BMC}$, research is always in progress.

Learn more biomedcentral.com/submission 